\title{
Hadronic total cross sections at high energy and the QCD spectrum
}

\author{
Matteo Giordano $^{a}$ and Enrico Meggiolaro ${ }^{b}$ \\ ${ }^{a}$ Institute for Nuclear Research of the Hungarian Academy of Sciences (ATOMKI), \\ Bem tér 18/c, H-4026 Debrecen, Hungary \\ ${ }^{b}$ Dipartimento di Fisica, Università di Pisa, and INFN, Sezione di Pisa, \\ Largo Pontecorvo 3, I-56127 Pisa, Italy \\ E-mail: giordano@atomki.mta.hu, enrico.meggiolaro@df.unipi.it
}

\begin{abstract}
We show how to obtain the leading energy dependence of hadronic total cross sections, in the framework of the nonperturbative approach to soft high-energy scattering based on Wilson-loop correlation functions, if certain nontrivial analyticity assumptions are satisfied. The total cross sections turn out to be of "Froissart" type, $\sigma_{\text {tot }}^{(h h)}(s) \sim B \log ^{2} s$ for $s \rightarrow \infty$. We also discuss under which conditions the coefficient $B$ is universal, i.e., independent of the hadrons involved in the scattering process. In the most natural scenarios for universality, $B$ can be related to the stable spectrum of QCD, and is predicted to be $B_{\text {th }} \simeq 0.22 \mathrm{mb}$, in fair agreement with experimental results. If we consider, instead, the stable spectrum of the quenched (i.e., pure-gauge) theory, we obtain a quite larger value $B_{\mathrm{th}}^{(Q)} \geq 0.42 \mathrm{mb}$, suggesting (quite surprisingly) large unquenching effects due to the sea quarks.
\end{abstract}

KEYwORDS: Nonperturbative Effects, QCD

ArXiv EPRINT: 1311.3133 


\section{Contents}

1 Introduction 1

2 Meson-meson scattering from dipole-dipole scattering $\quad 3$

3 Relating hadronic total cross sections and the QCD spectrum: outline 7

4 Wilson-loop correlation function and the hadronic spectrum 10

$\begin{array}{lll}4.1 & \text { Wilson loop in the operator formalism } & 10\end{array}$

$\begin{array}{lll}4.2 & \text { Inserting a complete set of states } & 11\end{array}$

$\begin{array}{lll}4.3 & \text { Analytic continuation to Minkowski space } & 15\end{array}$

$\begin{array}{lll}4.4 & \text { Large- } b \text { behaviour } & 18\end{array}$

$\begin{array}{lll}4.5 & \text { Large- } \chi \text { behaviour } & 19\end{array}$

$5 \quad$ Elastic scattering amplitude and total cross section $\quad 23$

5.1 Asymptotic behaviour of the total cross section 24

$\begin{array}{ll}5.2 & \text { Universality of total cross sections } \\ 5.3 & 26\end{array}$

$\begin{array}{lll}5.3 & \text { Elastic scattering amplitude at high energy } & 29\end{array}$

6 Conclusions 31

A Technical details $\quad \mathbf{3 4}$

A.1 Vanishing Wilson-loop matrix elements and massless particles 34

A.2 Resummation for factorised matrix elements: general case 37

\section{Introduction}

The recent measurements of proton-proton total cross sections at the LHC by the TOTEM collaboration [1-4], at total center-of-mass energy $\sqrt{s}=7 \mathrm{TeV}$ and $\sqrt{s}=8 \mathrm{TeV}$, have helped in reviving the study of the high-energy behaviour of hadronic total cross sections [58]. The main theoretical problem in this context is providing a convincing explanation of the rise with energy of total cross sections observed in experiments, and a definite prediction of its functional form, in the framework of QCD. Despite many years of efforts, a satisfactory solution to this problem is still lacking.

Experimental data for total cross sections are well described by a "Froissart-like" behaviour $\sigma_{\text {tot }}^{(h h)}(s) \sim B \log ^{2} s$ for $s \rightarrow \infty$, with a universal (i.e., not depending on the particular hadrons involved) coefficient $B \simeq 0.25-0.3 \mathrm{mb}$ [9-17]. The attribute "Froissart-like" is a reference to the functional form appearing in the well-known Froissart-Łukaszuk-Martin (FLM) bound [18-20], which states that for $s \rightarrow \infty, \sigma_{\text {tot }}^{(h h)}(s) \leq \frac{\pi}{m_{\pi}^{2}} \log ^{2}\left(\frac{s}{s_{0}}\right)$, where $m_{\pi}$ is 
the pion mass and $s_{0}$ is an unspecified squared mass scale. ${ }^{1}$ Theoretical supports to this functional form and to the universality of the coefficient $B$ were found in the model of the iteration of soft-Pomeron exchanges by eikonal unitarisation [25, 26] (recently revisited in the context of holographic QCD [27]), and also using arguments based on the so-called Color Glass Condensate of QCD [28, 29], or simply modifying the original Heisenberg's model [30] in connection with the presence of glueballs [31]. These arguments however do not provide a full derivation of the "Froissart-like" total cross sections from the first principles of QCD. We mention at this point that the $\log ^{2} s$ behaviour of total cross sections has been recently questioned in refs. [32-34], and the validity itself of the FLM bound has also been put under scrutiny [35] (see however also ref. [23] for a comment).

Explaining the behaviour of hadronic total cross sections is part of the more general problem of hadronic soft high-energy scattering, i.e., high-energy elastic scattering of hadrons at low transferred momentum. Soft high-energy processes are characterised by two different energy scales, provided by the total center-of-mass energy squared $s$, which is large, and the transferred momentum squared $t$, which is fixed and smaller than the typical (squared) energy scale of strong interactions $\left(|t| \lesssim 1 \mathrm{GeV}^{2} \ll s\right)$. As a consequence, the study of these processes cannot fully rely on perturbation theory. A nonperturbative approach to this problem in the framework of QCD has been proposed in ref. [36], and has been further developed in a number of papers (see, e.g., ref. [37] for a review and a complete list of references): using a functional integral approach, high-energy hadron-hadron elastic scattering amplitudes are shown to be governed by the correlation function of certain Wilson loops defined in Minkowski space [38-42]. Moreover, it has been shown in refs. [43-47] that this correlation function can be reconstructed by analytic continuation from the correlation function of two Euclidean Wilson loops. This has allowed the investigation of the correlators using the nonperturbative methods of Euclidean Field Theory, both through approximate analytical calculations in the Stochastic Vacuum Model (SVM) [48], in the Instanton Liquid Model (ILM) [49, 50], and using the AdS/CFT correspondence [51-54], and through numerical Monte Carlo simulations in Lattice Gauge Theory (LGT) [50, 55] (see also refs. $[56,57]$ for a short review).

As discussed in refs. [50, 55], the comparison of the analytic nonperturbative calculations in QCD-related models [48-50] (as well as that of the perturbative calculations $[46,48,58])$ to the numerical data from LGT is not satisfactory. As the numerical results obtained on the lattice can be considered "exact" (within the errors) predictions of QCD, this casts doubts on the viability of the above-mentioned models, which moreover do not lead to rising total cross sections.

Recently, a new analysis of the numerical results has been proposed in ref. [59]. The main purpose of that paper was to provide a parameterisation of the lattice data that, after analytic continuation to Minkowski space, results into a physically acceptable scattering amplitude satisfying the unitarity constraint, and that furthermore leads to a rising behaviour of total cross sections at high energy (beside, of course, fitting well the data). In

\footnotetext{
${ }^{1}$ Notice that the experimental value of $B$ is much smaller than the coefficient $\frac{\pi}{m_{\pi}^{2}}$ (about $0.5 \%$ ) appearing in the FLM bound. See refs. [21-23] for recent work to improve the bound, and also ref. [24].
} 
particular, we were able to identify and qualitatively justify a class of simple parameterisations that lead to universal "Froissart-like" behaviour. Moreover, the value of $B$ resulting from our fits was of the same order of magnitude of the experimental value, within the large errors, and notwithstanding the use of the quenched approximation in the numerical simulations. However, although the results above look promising, the functional forms used in the analysis of ref. [59] are not fully justified.

The purpose of this paper is to gain more insight both on the functional form of the relevant Wilson-loop correlators, and on the quantitative identification of its relevant parameters. The basic idea is to analyse the Euclidean correlators by inserting a complete set of states between the Wilson loops, and extracting the large impact-parameter behaviour of the Wilson-loop correlator. Under the assumption that the analytic continuation to Minkowski space can be performed term by term, we are able to identify the terms that dominate the sum at high energy, and in turn to compute the high-energy behaviour of total cross sections. Under the above-mentioned nontrivial analyticity assumption, we provide a derivation of the "Froissart-like" behaviour of hadronic total cross sections in the framework of QCD. Furthermore, we discuss how one can obtain universality of this behaviour, and how the coefficient $B$ of the $\log ^{2} s$ term is related to the hadronic spectrum.

The plan of the paper is the following. In section 2 we give a brief account of the nonperturbative approach to soft high-energy scattering, based on the correlation function of Wilson loops in the sense of the QCD functional integral. We also discuss the issue of analytic continuation to Euclidean space. In section 3 we give a general outline of our argument, to provide a guide for the reader to the more technical discussion of the following sections. In section 4 we relate the functional-integral language with the operator language, and we re-express the Wilson-loop correlation function in terms of a sum over a complete set of states. After performing the analytic continuation to Minkowski space, we investigate the limits of large energy and large impact parameter. In section 5 we use the corresponding results to investigate the high-energy behaviour of the hadronic total cross sections and of the elastic scattering amplitudes. Finally, in section 6 we draw our conclusions. Some technical details are discussed in appendix A.

\section{Meson-meson scattering from dipole-dipole scattering}

In this section we briefly sketch the nonperturbative approach to soft high-energy scattering (see ref. [55] for a more detailed presentation, and also ref. [60] for a recent re-derivation of the main formula). The elastic scattering amplitude $\mathcal{M}_{(h h)}$ of two hadrons, or more precisely mesons (taken for simplicity with the same mass $m$ ), in the soft high-energy regime can be reconstructed from the scattering amplitude $\mathcal{M}_{(d d)}$ of two dipoles of fixed transverse sizes $\vec{R}_{1,2 \perp}$, and fixed longitudinal-momentum fractions $f_{1,2}$ of the two quarks in the two dipoles, after folding with two proper squared hadron wave functions $\left|\psi_{1}\right|^{2}$ and $\left|\psi_{2}\right|^{2}$, describing the two interacting hadrons [38-42]:

$$
\begin{aligned}
\mathcal{M}_{(h h)}(s, t)= & \int d^{2} \vec{R}_{1 \perp} \int_{0}^{1} d f_{1}\left|\psi_{1}\left(\vec{R}_{1 \perp}, f_{1}\right)\right|^{2} \int d^{2} \vec{R}_{2 \perp} \int_{0}^{1} d f_{2}\left|\psi_{2}\left(\vec{R}_{2 \perp}, f_{2}\right)\right|^{2} \\
& \times \mathcal{M}_{(d d)}\left(s, t ; \vec{R}_{1 \perp}, f_{1}, \vec{R}_{2 \perp}, f_{2}\right) \equiv\left\langle\left\langle\mathcal{M}_{(d d)}\left(s, t ; \nu_{1}, \nu_{2}\right)\right\rangle\right\rangle
\end{aligned}
$$




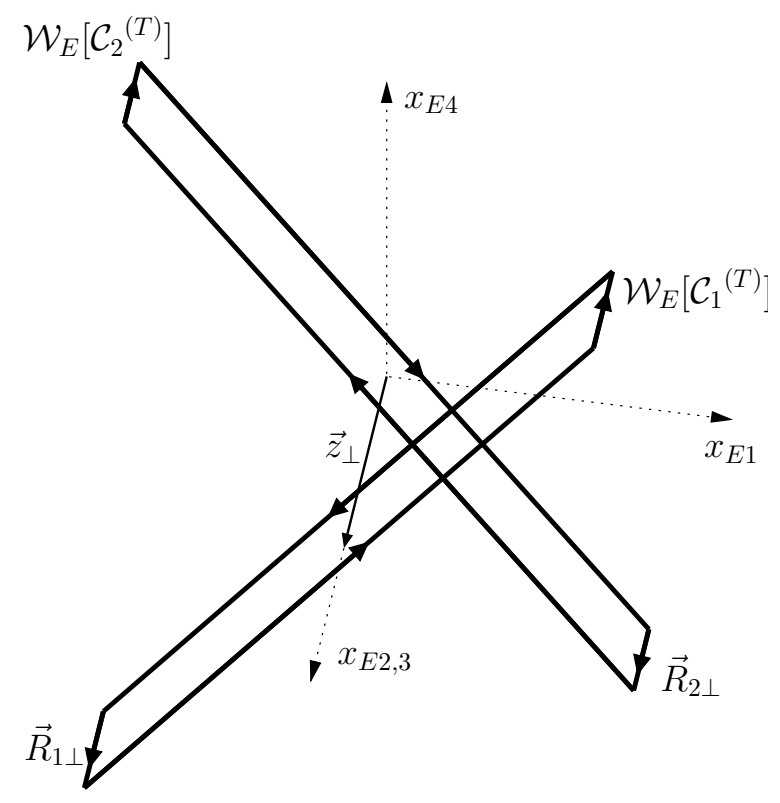

Figure 1. The relevant Wilson loops in Euclidean space.

with: $\int d^{2} \vec{R}_{1 \perp} \int_{0}^{1} d f_{1}\left|\psi_{1}\left(\vec{R}_{1 \perp}, f_{1}\right)\right|^{2}=\int d^{2} \vec{R}_{2 \perp} \int_{0}^{1} d f_{2}\left|\psi_{2}\left(\vec{R}_{2 \perp}, f_{2}\right)\right|^{2}=1$, so that $\langle\langle 1\rangle\rangle=1$. The notation $\nu_{i}=\left(\vec{R}_{i \perp}, f_{i}\right)$ will be often used for the sake of brevity. For the treatment of baryons, a similar picture can be adopted, using a genuine three-body configuration or, alternatively and even more simply, a quark-diquark configuration: we refer the interested reader to the above-mentioned original references [38-42] and to ref. [61].

In turn, the dipole-dipole scattering amplitude is obtained from the (properly normalised) correlation function of two Wilson loops in the fundamental representation, defined in Minkowski spacetime, running along the paths made up of the quark and antiquark classical straight-line trajectories, and thus forming a hyperbolic angle $\chi \simeq \log \left(s / \mathrm{m}^{2}\right)$ in the longitudinal plane. The paths are cut at proper times $\pm T$ as an infrared regularisation, and closed by straight-line "links" in the transverse plane, in order to ensure gauge invariance. Eventually, the limit $T \rightarrow \infty$ has to be taken. It has been shown in refs. [43-47] that the relevant Minkowskian correlation function $\mathcal{G}_{M}\left(\chi ; T ; \vec{z}_{\perp} ; \nu_{1}, \nu_{2}\right)\left(\vec{z}_{\perp}\right.$ being the impact parameter, i.e., the transverse separation between the two dipoles) can be reconstructed, by means of analytic continuation, from the Euclidean correlation function of two Euclidean Wilson loops,

$$
\mathcal{G}_{E}\left(\theta ; T ; \vec{z}_{\perp} ; \nu_{1}, \nu_{2}\right) \equiv \frac{\left\langle\mathcal{W}_{E}\left[\mathcal{C}_{1}^{(T)}\right] \mathcal{W}_{E}\left[\mathcal{C}_{2}^{(T)}\right]\right\rangle_{E}}{\left\langle\mathcal{W}_{E}\left[\mathcal{C}_{1}^{(T)}\right]\right\rangle_{E}\left\langle\mathcal{W}_{E}\left[\mathcal{C}_{2}^{(T)}\right]\right\rangle_{E}}-1,
$$

where $\langle\ldots\rangle_{E}$ is the average in the sense of the Euclidean QCD functional integral. The Euclidean Wilson loop is defined as follows,

$$
\mathcal{W}_{E}[\mathcal{C}] \equiv \frac{1}{N_{c}} \operatorname{Tr} \operatorname{Pexp}\left\{-i g \oint_{\mathcal{C}} A_{E \mu}\left(x_{E}\right) d x_{E \mu}\right\},
$$

where $P$ stands for path-ordering with larger values of the path parameter appearing on 
the left. ${ }^{2}$ The Wilson loops appearing in eq. (2.2) are computed on the paths made up of the following quark $[+]$ - antiquark [-] straight-line paths (see figure 1),

$$
\mathcal{C}_{1}^{(T)}: X_{E 1}^{ \pm}(\tau)= \pm u_{1} \tau+z+f_{1}^{ \pm} R_{1}, \quad \mathcal{C}_{2}^{(T)}: X_{E 2}^{ \pm}(\tau)= \pm u_{2} \tau+f_{2}^{ \pm} R_{2},
$$

with $\tau \in[-T, T]$, and closed by straight-line paths in the transverse plane at $\tau= \pm T$. The four-vectors $u_{1,2}$ are chosen to be $u_{1,2}=\left( \pm \sin \frac{\theta}{2}, \overrightarrow{0}_{\perp}, \cos \frac{\theta}{2}\right), \theta$ being the angle formed by the two trajectories, i.e., $u_{1} \cdot u_{2}=\cos \theta$. Moreover, $R_{i}=\left(0, \vec{R}_{i \perp}, 0\right), z=\left(0, \vec{z}_{\perp}, 0\right)$ and $f_{i}^{+} \equiv 1-f_{i}, f_{i}^{-} \equiv-f_{i}$. We define also the Euclidean and Minkowskian correlation functions with the infrared cutoff removed as

$$
\begin{aligned}
\mathcal{C}_{E}\left(\theta ; \vec{z}_{\perp} ; \nu_{1}, \nu_{2}\right) & \equiv \lim _{T \rightarrow \infty} \mathcal{G}_{E}\left(\theta ; T ; \vec{z}_{\perp} ; \nu_{1}, \nu_{2}\right), \\
\mathcal{C}_{M}\left(\chi ; \vec{z}_{\perp} ; \nu_{1}, \nu_{2}\right) & \equiv \lim _{T \rightarrow \infty} \mathcal{G}_{M}\left(\chi ; T ; \vec{z}_{\perp} ; \nu_{1}, \nu_{2}\right)
\end{aligned}
$$

The dipole-dipole scattering amplitude is then obtained from $\mathcal{C}_{E}(\theta ; \ldots)$, with $\theta \in(0, \pi)$, by means of analytic continuation as

$$
\begin{aligned}
\mathcal{M}_{(d d)}\left(s, t ; \nu_{1}, \nu_{2}\right) & \equiv-i 2 s \int d^{2} \vec{z}_{\perp} e^{i \vec{q}_{\perp} \cdot \vec{z}_{\perp}} \mathcal{C}_{M}\left(\chi \simeq \log \left(s / m^{2}\right) ; \vec{z}_{\perp} ; \nu_{1}, \nu_{2}\right) \\
& =-i 2 s \int d^{2} \vec{z}_{\perp} e^{i \vec{q}_{\perp} \cdot \vec{z}_{\perp}} \mathcal{C}_{E}\left(\theta \rightarrow-i \chi \simeq-i \log \left(s / m^{2}\right) ; \vec{z}_{\perp} ; \nu_{1}, \nu_{2}\right)
\end{aligned}
$$

with $\chi \in \mathbb{R}^{+}$, and where $s$ and $t=-\left|\vec{q}_{\perp}\right|^{2}$ ( $\vec{q}_{\perp}$ being the transferred momentum) are the usual Mandelstam variables (for a detailed discussion on the analytic continuation see ref. [47], where we have shown, on nonperturbative grounds, that the required analyticity hypotheses are indeed satisfied). The restrictions on the domains of the variables $\theta$ and $\chi$ cause no loss of generality, due to the symmetries of the Euclidean and Minkowskian theories $[62,63]$.

For our purposes, it is convenient to exploit the rotation invariance of the Minkowskian theory in order to fix the direction of $\vec{z}_{\perp}$ along, say, the 2-axis. Indeed, dropping all the variables that are irrelevant here, and choosing a rotation of angle $\varphi$ around the 1-axis in such a way that $\mathcal{R}_{\varphi} \vec{b}_{\perp}=\vec{z}_{\perp},{ }^{3}$ with $\vec{b}_{\perp}=(b, 0)$ and $b=\left|\vec{z}_{\perp}\right|$, we have

$$
\begin{aligned}
& \int d^{2} \vec{R}_{1 \perp}\left|\psi_{1}\left(\vec{R}_{1 \perp}\right)\right|^{2} \int d^{2} \vec{R}_{2 \perp}\left|\psi_{2}\left(\vec{R}_{2 \perp}\right)\right|^{2} \int d^{2} \vec{z}_{\perp} e^{i \vec{q}_{\perp} \cdot \vec{z}_{\perp}} \mathcal{C}_{M}\left(\vec{z}_{\perp} ; \vec{R}_{1 \perp}, \vec{R}_{2 \perp}\right) \\
= & \int d^{2} \vec{z}_{\perp} e^{i \vec{q}_{\perp} \cdot \vec{z}_{\perp}} \int d^{2} \vec{R}_{1 \perp}\left|\psi_{1}\left(\mathcal{R}_{\varphi} \vec{R}_{1 \perp}\right)\right|^{2} \int d^{2} \vec{R}_{2 \perp}\left|\psi_{2}\left(\mathcal{R}_{\varphi} \vec{R}_{2 \perp}\right)\right|^{2} \mathcal{C}_{M}\left(\vec{b}_{\perp} ; \vec{R}_{1 \perp}, \vec{R}_{2 \perp}\right),
\end{aligned}
$$

and we can write $\int d^{2} \vec{z}_{\perp}=\int_{0}^{\infty} d b b \int_{0}^{2 \pi} d \varphi$. Expression eq. (2.7) simplifies in two cases. If $\vec{q}_{\perp}=0$, the only dependence on the orientation of $\vec{z}_{\perp}$ appears in the wave functions, so

\footnotetext{
${ }^{2}$ Usually, path-ordering requires larger values of the path parameter to appear on the right, while our definition of path-ordering is usually called time-ordering and is denoted with $T$. The usual convention has been followed in our previous papers. However, here we will also use the time-ordered product of operators, for which we have preferred to reserve the symbol $T$.

${ }^{3}$ Here $\mathcal{R}_{\varphi}$ denotes the restriction of the rotation to the $(2,3)$-plane.
} 


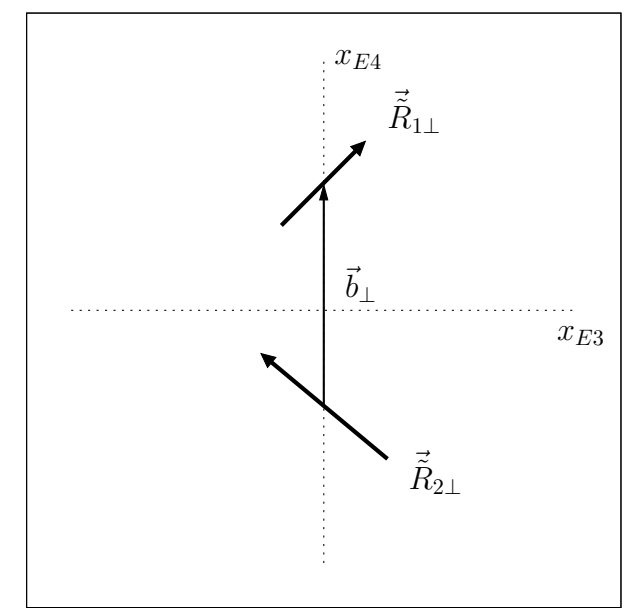

Figure 2. Relevant configuration of dipoles in Euclidean space after aligning the impact parameter with Euclidean "time".

that we can treat the angular integration over $\varphi$ as part of the averaging over the dipole variables, i.e., we can write

$$
\begin{aligned}
\mathcal{M}_{(h h)}(s, 0)= & -4 \pi i s \int_{0}^{2 \pi} \frac{d \varphi}{2 \pi} \int d^{2} \vec{R}_{1 \perp}\left|\psi_{1}\left(\mathcal{R}_{\varphi} \vec{R}_{1 \perp}\right)\right|^{2} \int d^{2} \vec{R}_{2 \perp}\left|\psi_{2}\left(\mathcal{R}_{\varphi} \vec{R}_{2 \perp}\right)\right|^{2} \\
& \times \int_{0}^{\infty} d b b \mathcal{C}_{M}\left(\vec{b}_{\perp} ; \vec{R}_{1 \perp}, \vec{R}_{2 \perp}\right) \equiv-4 \pi i s\left\langle\left\langle\int_{0}^{\infty} d b b \mathcal{C}_{M}\left(\vec{b}_{\perp} ; \vec{R}_{1 \perp}, \vec{R}_{2 \perp}\right)\right\rangle\right\rangle_{\varphi}
\end{aligned}
$$

where $\langle\langle 1\rangle\rangle_{\varphi}=1$. Notice that we are not making any assumption on the wave functions. The other case is that in which the wave functions are independent of the orientation of the dipoles: this is the case, for example, if one considers amplitudes for unpolarised scattering. Under this condition, $\mathcal{R}_{\varphi}$ drops from the wave functions in eq. (2.7), and after carrying out the integration over $\varphi$ one obtains the following simple form for the meson-meson scattering amplitude,

$$
\mathcal{M}_{(h h)}(s, t)=-4 \pi i s\left\langle\left\langle\int_{0}^{\infty} d b b J_{0}(b \sqrt{-t}) \mathcal{C}_{M}\left(\chi \simeq \log \left(s / m^{2}\right) ; \vec{b}_{\perp} ; \nu_{1}, \nu_{2}\right)\right\rangle\right\rangle_{0}
$$

where by the subscript 0 we indicate explicitly that the wave functions are rotationinvariant. We note here that in most phenomenological applications of the nonperturbative approach to soft high energy scattering, the hadron wave functions are chosen to be invariant under rotations and under the exchange $f_{i} \rightarrow 1-f_{i}$ (see refs. [41, 42] and also [37], section 8.6, and references therein).

Clearly, $\mathcal{C}_{M}\left(\chi ; \vec{b}_{\perp} ; \nu_{1}, \nu_{2}\right)=\mathcal{C}_{E}\left(\theta \rightarrow-i \chi ; \vec{b}_{\perp} ; \nu_{1}, \nu_{2}\right)$, due to the analytic continuation relations. Furthermore, we can exploit the $O(4)$ invariance of the Euclidean theory to show that

$$
\mathcal{C}_{E}\left(\theta ; \vec{b}_{\perp} ; \nu_{1}, \nu_{2}\right)=\tilde{\mathcal{C}}_{E}\left(\theta ; b ; \nu_{1}, \nu_{2}\right)=\lim _{T \rightarrow \infty} \tilde{\mathcal{G}}_{E}\left(\theta ; T ; b ; \nu_{1}, \nu_{2}\right)
$$


where $\tilde{\mathcal{G}}_{E}$ is the correlation function of two Wilson loops computed on new paths $\tilde{\mathcal{C}}_{1,2}^{(T)}$,

$$
\tilde{\mathcal{G}}_{E}\left(\theta ; T ; b ; \nu_{1}, \nu_{2}\right) \equiv \frac{\left\langle\mathcal{W}_{E}\left[\tilde{\mathcal{C}}_{1}^{(T)}\right] \mathcal{W}_{E}\left[\tilde{\mathcal{C}}_{2}^{(T)}\right]\right\rangle_{E}}{\left\langle\mathcal{W}_{E}\left[\tilde{\mathcal{C}}_{1}^{(T)}\right]\right\rangle_{E}\left\langle\mathcal{W}_{E}\left[\tilde{\mathcal{C}}_{2}^{(T)}\right]\right\rangle_{E}}-1
$$

with $\tilde{\mathcal{C}}_{1,2}^{(T)}$ obtained from $\mathcal{C}_{1,2}^{(T)}$ by rotating the transverse separation $\left(0, \vec{b}_{\perp}, 0\right)$ along the Euclidean "time" direction $x_{E 4}$, see figure 2. Explicitly, these paths are defined by

$$
\tilde{\mathcal{C}}_{1}^{(T)}: \tilde{X}_{E 1}^{ \pm}(\tau)= \pm \tilde{u}_{1} \tau+\tilde{z}+f_{1}^{ \pm} \tilde{R}_{1}, \quad \tilde{\mathcal{C}}_{2}^{(T)}: \tilde{X}_{E 2}^{ \pm}(\tau)= \pm \tilde{u}_{2} \tau+f_{2}^{ \pm} \tilde{R}_{2},
$$

with $\tau \in[-T, T]$, and closed by appropriate straight-line paths at $\tau= \pm T$. Here

$$
\begin{aligned}
\tilde{u}_{1,2} & =\left(\cos \frac{\theta}{2}, \pm \sin \frac{\theta}{2}, 0,0\right), & \tilde{z}=(0,0,0, b), \\
\tilde{R}_{i} & =\left(0,0, \overrightarrow{\tilde{R}}_{i \perp}\right)=\left(0,0, r_{i} \sin \phi_{i}, r_{i} \cos \phi_{i}\right), & i=1,2,
\end{aligned}
$$

where $r_{i}=\left|\vec{R}_{i \perp}\right|, \phi_{i}$ is the angle formed by $\vec{b}_{\perp}$ and $\vec{R}_{i \perp}$, and $f_{i}^{ \pm}$have been defined after eq. (2.4). For future utility, we define also the paths $\tilde{\mathcal{C}}_{0}^{(T)}\left(\nu_{i}\right)$,

$$
\tilde{\mathcal{C}}_{0}^{(T)}\left(\nu_{i}\right): \tilde{X}_{E 0}^{ \pm}(\tau)= \pm \tilde{u}_{0} \tau+f_{i}^{ \pm} \tilde{R}_{i}, \quad u_{0}=(1,0,0,0)
$$

again with $\tau \in[-T, T]$, and closed by appropriate straight-line paths. These are nothing but rectangular paths centered ${ }^{4}$ at the origin, and with the "long" side parallel to direction 1. Obviously, $\mathcal{C}_{M}\left(\chi ; \vec{b}_{\perp} ; \nu_{1}, \nu_{2}\right)=\tilde{\mathcal{C}}_{E}\left(\theta \rightarrow-i \chi ; b ; \nu_{1}, \nu_{2}\right)$, so that $\tilde{\mathcal{C}}_{E}$ encodes all the relevant information on the scattering amplitude.

We finally mention that the amplitude for meson-antimeson scattering is obtained from eq. (2.6) by replacing $\vec{R}_{2 \perp}, f_{2} \rightarrow-\vec{R}_{2 \perp}, 1-f_{2}$, or equivalently by performing the analytic continuation $\chi \rightarrow i \pi-\chi$ of the Minkowskian correlator $\mathcal{C}_{M}$, thanks to the crossingsymmetry relations discussed in refs. [62, 63],

$$
\mathcal{C}_{M}\left(\chi ; \vec{z}_{\perp} ; \nu_{1}, \bar{\nu}_{2}\right)=\mathcal{C}_{M}\left(\chi ; \vec{z}_{\perp} ; \bar{\nu}_{1}, \nu_{2}\right)=\mathcal{C}_{M}\left(i \pi-\chi ; \vec{z}_{\perp} ; \nu_{1}, \nu_{2}\right),
$$

where $\bar{\nu}_{i}=\left(-\vec{R}_{i \perp}, 1-f_{i}\right)$. Notice that for hadronic wave functions invariant under rotations and under the exchange $f_{i} \rightarrow 1-f_{i}$ [see after eq. (2.9)], the scattering amplitude is automatically crossing-symmetric.

\section{Relating hadronic total cross sections and the QCD spectrum: outline}

As we have stated in the Introduction, the purpose of this paper is to obtain new insights on the high-energy behaviour of hadronic total cross sections, by relating the Wilson-loop correlation functions from which the scattering amplitudes are built in the soft high energy regime to the spectrum of QCD. As this involves a certain number of rather technical steps, we want to provide first a general outline of our argument, to make it easier for the reader to follow the detailed discussion of the following sections.

\footnotetext{
${ }^{4}$ Here by "center" of the loop we mean the "center of mass" of the dipole at $\tau=0$, i.e., $f_{i} X_{E 0}^{+}(0)+(1-$ $\left.f_{i}\right) X_{E 0}^{-}(0)$.
} 
The starting point is to re-express the relevant Euclidean correlation function in the operator language. This requires the introduction of the Euclidean Wilson loop operator $\hat{\mathcal{W}}_{E}$, which will be defined precisely in eq. (4.4) below. In terms of $\hat{\mathcal{W}}_{E}$, Wilson-loop correlation functions in the functional-integral formalism are rewritten as vacuum expectation values of $T$-ordered products of Wilson loop operators. For our purposes, it is convenient to work with the correlation function $\tilde{\mathcal{G}}_{E}$ defined in eq. (2.11), for which the separation along Euclidean "time" is equal to the impact-parameter distance $b$ in the scattering process. For sufficiently large $b$, so that there are no (Euclidean) time-ordering issues [see eq. (4.7)], the relevant correlator reads (up to normalisation factors)

$$
\left\langle\mathcal{W}_{E}\left[\tilde{\mathcal{C}}_{1}^{(T)}\right] \mathcal{W}_{E}\left[\tilde{\mathcal{C}}_{2}^{(T)}\right]\right\rangle_{E}=\left\langle 0\left|\hat{\mathcal{W}}_{E}\left[\tilde{\mathcal{C}}_{1}^{(T)}\right] \hat{\mathcal{W}}_{E}\left[\tilde{\mathcal{C}}_{2}^{(T)}\right]\right| 0\right\rangle
$$

where the paths $\tilde{\mathcal{C}}_{1,2}^{(T)}$ have been defined above in eq. (2.12).

The form eq. (3.1) of the correlation function is suitable for inserting a complete set of states between the two Wilson loops. For this purpose we use asymptotic states characterised by their particle content, and by the momentum and third component of the spin of each particle. Denoting by $\alpha$ a generic state, and exploiting the Euclidean symmetries, one finds

$$
\begin{aligned}
\left\langle\mathcal{W}_{E}\left[\tilde{\mathcal{C}}_{1}^{(T)}\right] \mathcal{W}_{E}\left[\tilde{\mathcal{C}}_{2}^{(T)}\right]\right\rangle_{E} & =\sum_{\alpha}\left\langle 0\left|\hat{\mathcal{W}}_{E}\left[\tilde{\mathcal{C}}_{1}^{(T)}\right]\right| \alpha\right\rangle\left\langle\alpha\left|\hat{\mathcal{W}}_{E}\left[\tilde{\mathcal{C}}_{2}^{(T)}\right]\right| 0\right\rangle \\
& =\sum_{\alpha} e^{-b E_{\alpha}} e^{i \theta S_{3}^{(\alpha)}}\left\langle 0\left|\hat{\mathcal{W}}_{E}\left[\tilde{\mathcal{C}}_{0}^{(T)}\left(\nu_{1}\right)\right]\right| \alpha_{\frac{\theta}{2}}\right\rangle\left\langle\alpha_{-\frac{\theta}{2}}\left|\hat{\mathcal{W}}_{E}\left[\tilde{\mathcal{C}}_{0}^{(T)}\left(\nu_{2}\right)\right]\right| 0\right\rangle
\end{aligned}
$$

where the sum over $\alpha$ includes also the appropriate phase-space integration over the particles' momenta. Here $E_{\alpha}$ and $S_{3}^{(\alpha)}$ are the total energy and total third component of the spin for state $\alpha$, respectively, and the paths $\tilde{\mathcal{C}}_{0}^{(T)}\left(\nu_{1,2}\right)$, which have been defined in eq. (2.14), are independent of $b$ and $\theta$. Moreover, the states $\left|\alpha_{ \pm \frac{\theta}{2}}\right\rangle$ are obtained from $|\alpha\rangle$ by performing a rotation of the momenta of $\pm \frac{\theta}{2}$ around the third axis [see eq. (4.19)]. The use of time-translation invariance in the second line of eq. (3.2) allows to completely expose the dependence on $b$, while the use of rotation invariance allows to shift the dependence on $\theta$ from the loops to the momenta of the particles in the intermediate states; a further simple change of variables allows to expose the $\theta$-dependence almost entirely [see eqs. (4.20)-(4.22)], yielding

$$
\begin{aligned}
\tilde{\mathcal{C}}_{E} & =\lim _{T \rightarrow \infty} \frac{\left\langle 0\left|\mathcal{W}_{E}\left[\tilde{\mathcal{C}}_{1}^{(T)}\right] \mathcal{W}_{E}\left[\tilde{\mathcal{C}}_{2}^{(T)}\right]\right| 0\right\rangle}{\left\langle 0\left|\hat{\mathcal{W}}_{E}\left[\tilde{\mathcal{C}}_{1}^{(T)}\right]\right| 0\right\rangle\left\langle 0\left|\hat{\mathcal{W}}_{E}\left[\tilde{\mathcal{C}}_{2}^{(T)}\right]\right| 0\right\rangle}-1 \\
& =\sum_{\alpha \neq 0} \frac{e^{-b E_{\alpha}} e^{i \theta \theta_{3}^{(\alpha)}}}{(\sin \theta)^{\mathcal{N}_{\alpha}}} M_{\alpha}\left(\theta ; \nu_{1}, \nu_{2}\right)=\sum_{\alpha \neq 0} \delta C_{\alpha},
\end{aligned}
$$

where $\mathcal{N}_{\alpha}$ is the number of particles in state $\alpha, M_{\alpha}$ is given by the Wilson-loop matrix elements expressed in terms of the new variables [times appropriate phase-space factors, see eq. (4.26)], and we have taken the physical limit $T \rightarrow \infty$ [see eqs. (2.10) and (4.28)].

The next step is to perform the analytic continuation to Minkowski space, which for the correlation function $\tilde{\mathcal{C}}_{E}$ reduces to taking $\theta \rightarrow-i \chi$, and to study the large- $\chi$ limit. 
To this extent, we make the crucial assumption that the analytic continuation can be carried out term by term, i.e., we assume that the analytic continuation can be performed independently for each term $\delta C_{\alpha}$ in the sum over states in eq. (3.3). This requires that the sum has "good" convergence properties (e.g., uniform convergence in $\theta$ ), so that analytic continuation and summation commute. Under this assumption, it is easy to carry out the analytic continuation, and to determine separately the leading energy dependence of each term in the sum in the physical limit of large $\chi \sim \log s$. This is due to the fact that, after analytic continuation, the function $M_{\alpha}\left(\theta \rightarrow-i \chi ; \nu_{1}, \nu_{2}\right)$ in eq. (3.3) becomes independent of $\chi$ at large $\chi$ [see eqs. (4.31)-(4.35)]. Assuming that it is a finite nonzero quantity (see however footnote 11), it is therefore possible to read off the leading power of $s \sim e^{\chi}$ for each contribution directly from eq. (3.3). One can easily see that at fixed particle content the dominant contributions come from states with maximal total spin. Furthermore, it is clear from eq. (3.3) that at large $b$ each contribution dies off exponentially. More precisely, for maximal total spin the leading term in $\delta C_{\alpha}$ receives a factor $e^{\chi\left[s^{(a)}-1\right]} e^{-b m^{(a)}}$ from each particle, i.e., up to $\chi, b$-independent factors and inverse powers of $b$ one finds

$$
\delta C_{\alpha} \sim \prod_{a}\left[e^{\chi\left[s^{(a)}-1\right]} e^{-b m^{(a)}}\right]^{n_{a}(\alpha)}
$$

with $m^{(a)}$ and $s^{(a)}$ respectively the mass and spin of particles of type $a$, and $n_{a}(\alpha)$ the corresponding occupation number in state $\alpha$. In physical terms, this means that states containing only particles of type $a$ contribute appreciably to the correlator only up to impact-parameter distances of the order of the "effective radius" $R_{\mathrm{eff}}^{(a)}=\chi\left[s^{(a)}-1\right] / m^{(a)}$ [or of an appropriate weighted average of the effective radii, if different species of particles are present, see eq. (4.40)].

The final step consists in realising that, for our purposes, the relevant contributions to the Wilson-loop correlator come from states containing only a single type of particles, namely those with maximal "effective radius". This is because to obtain the elastic scattering amplitude and the total cross section one has to integrate over the impact parameter, and the dominant contributions to the integrals in the large- $\chi$ limit come precisely from particles with maximal "effective radius". In turn, this implies that the leading relevant contributions to the correlator depend on $b$ only through the combination $z=z(\chi, b)=e^{\chi(\tilde{s}-1)} e^{-b \tilde{m}}$, where $\tilde{m}$ and $\tilde{s}$ are respectively the mass and spin of the particle maximising the ratio $\left(s^{(a)}-1\right) / m^{(a)}$. More precisely, up to constant factors, each of the $n$-particle sectors contributes a term proportional to $w^{n}$, where $w=w(\chi, z(\chi, b)) \propto z / \chi^{\lambda}$ for some real $\lambda$, whose precise value turns out to be irrelevant for the leading asymptotic behaviour of the total cross section.

Summarising, under the analyticity and finiteness assumptions mentioned above, it is possible to show that at large $\chi$ the relevant (Minkowskian) Wilson-loop correlation function, $\mathcal{C}_{M}\left(\chi ; \vec{b}_{\perp} ; \nu_{1}, \nu_{2}\right)$, depends only on a specific combination, $w$, of $\chi$ and $b$, i.e.,

$$
\mathcal{C}_{M}\left(\chi ; \vec{b}_{\perp} ; \nu_{1}, \nu_{2}\right) \underset{s \rightarrow \infty}{\sim} g\left(w ; \nu_{1}, \nu_{2}\right)-1
$$

[see eq. (4.49)] for sufficiently large $b$ [see eq. (4.7)]. Furthermore, one finds that the 
relevant features of the detailed form of $w$ depend only on the spectrum of the theory. This constitutes the first part of our program, and will be discussed in section 4 .

Having derived the large- $\chi$ behaviour of the relevant Wilson-loop correlation function, one can study the consequences for the asymptotic behaviour of hadronic total cross sections. An essential ingredient here is the unitarity constraint, which provides bounds on the scattering amplitude in impact-parameter space. As we argue in section 5, the unitarity constraint translates into a bound on the relevant Minkowskian correlator, i.e., $\left|\mathcal{C}_{M}\left(\chi ; \vec{b}_{\perp} ; \nu_{1}, \nu_{2}\right)+1\right| \leq 1$, which in turn implies that $g$ in eq. (3.5) is bounded [see eq. (5.6)]. This immediately allows to identify the large- $b$ region as the one giving the dominant contribution to the total cross sections, and to obtain a "Froissart-like" bound on the total cross sections [see eq. (5.22)],

$$
\sigma_{\text {tot }}^{(h h)}(s) \underset{s \rightarrow \infty}{\lesssim} 4 \pi \frac{(\tilde{s}-1)^{2}}{\tilde{m}^{2}}\left(\log \frac{s}{m^{2}}\right)^{2},
$$

where $\tilde{s}$ and $\tilde{m}$ have been defined above. If $g(w)$ is either vanishing or oscillating at large $w$, one can derive a stronger result, namely one can predict the asymptotic behaviour of $\sigma_{\text {tot }}^{(h h)}$ and show that it is universal, i.e., independent of the kind of hadrons involved. Explicitly, one finds [see eq. (5.34)]

$$
\sigma_{\text {tot }}^{(h h)}(s) \underset{s \rightarrow \infty}{\simeq} 2 \pi \frac{(\tilde{s}-1)^{2}}{\tilde{m}^{2}}\left(\log \frac{s}{m^{2}}\right)^{2} .
$$

Remarkably, in this case the prefactor of $\log ^{2} s$ is shown to be entirely determined by the spectrum of QCD, and can be predicted by finding the type of particle with maximal effective radius, as explained above. The detailed discussion of these issues, and a few results on the elastic scattering amplitudes, are reported in section 5 .

\section{Wilson-loop correlation function and the hadronic spectrum}

In this section we will show how the relevant Wilson loop correlator can be related to the QCD spectrum, discussing in full detail the first part of the argument outlined above in section 3. The consequences of our results for the hadronic total cross sections and elastic scattering amplitudes will be discussed in section 5 .

\subsection{Wilson loop in the operator formalism}

The "good" definition of the Wilson loop operator in Minkowski space, preserving its gauge invariance, is the following [64]:

$$
\hat{\mathcal{W}}[\mathcal{C}] \equiv \frac{1}{N_{c}} \operatorname{Tr} T P \exp \left\{-i g \oint_{\mathcal{C}} \hat{A}_{\mu}(x) d x^{\mu}\right\}
$$

Here and in the following the "hat" denotes an operator, and TP stands for both timeordering, acting on operators, and path-ordering, acting on the colour matrices. Explicitly,

$$
\begin{aligned}
T P & \left\{\hat{A}_{\mu}(x(\tau)) \hat{A}_{\nu}\left(x\left(\tau^{\prime}\right)\right)\right\}=\left\{\Theta\left(x^{0}(\tau)-x^{0}\left(\tau^{\prime}\right)\right) \hat{A}_{\mu}^{a}(x(\tau)) \hat{A}_{\nu}^{b}\left(x\left(\tau^{\prime}\right)\right)\right. \\
& \left.+\Theta\left(x^{0}\left(\tau^{\prime}\right)-x^{0}(\tau)\right) \hat{A}_{\nu}^{b}\left(x\left(\tau^{\prime}\right)\right) \hat{A}_{\mu}^{a}(x(\tau))\right\}\left\{\Theta\left(\tau-\tau^{\prime}\right) t^{a} t^{b}+\Theta\left(\tau^{\prime}-\tau\right) t^{b} t^{a}\right\}
\end{aligned}
$$


where $\Theta(x)$ is the Heaviside step function, and similarly in the case of more terms. The bridge between the operator formalism and the functional-integral formalism is provided by the relation

$$
\left\langle\mathcal{W}\left[\mathcal{C}_{1}\right] \ldots \mathcal{W}\left[\mathcal{C}_{n}\right]\right\rangle=\left\langle 0\left|T\left\{\hat{\mathcal{W}}\left[\mathcal{C}_{1}\right] \ldots \hat{\mathcal{W}}\left[\mathcal{C}_{n}\right]\right\}\right| 0\right\rangle,
$$

where the time ordering is understood to act on the expansion of the Wilson loops in products of field operators. The definition of the Euclidean Wilson loop is the same as in eq. (4.1),

$$
\hat{\mathcal{W}}_{E}[\mathcal{C}] \equiv \frac{1}{N_{c}} \operatorname{Tr} T P \exp \left\{-i g \oint_{\mathcal{C}} \hat{A}_{E \mu}\left(x_{E \mu}\right) d x_{E \mu}\right\},
$$

the only difference being that now the time-ordering is done with respect to the Euclidean "time". Also,

$$
\left\langle\mathcal{W}_{E}\left[\mathcal{C}_{1}\right] \ldots \mathcal{W}_{E}\left[\mathcal{C}_{n}\right]\right\rangle_{E}=\left\langle 0\left|T\left\{\hat{\mathcal{W}}_{E}\left[\mathcal{C}_{1}\right] \ldots \hat{\mathcal{W}}_{E}\left[\mathcal{C}_{n}\right]\right\}\right| 0\right\rangle,
$$

where $T$ is again time-ordering with respect to the Euclidean "time".

Let us now focus on the case of interest. Using eq. (4.5), we can recast the correlation function eq. (2.11) in terms of vacuum expectation values as follows,

$$
\tilde{\mathcal{G}}_{E}\left(\theta ; T ; b ; \nu_{1}, \nu_{2}\right)=\frac{\left\langle 0\left|T\left\{\hat{\mathcal{W}}_{E}\left[\tilde{\mathcal{C}}_{1}^{(T)}\right] \hat{\mathcal{W}}_{E}\left[\tilde{\mathcal{C}}_{2}^{(T)}\right]\right\}\right| 0\right\rangle}{\left\langle 0\left|\hat{\mathcal{W}}_{E}\left[\tilde{\mathcal{C}}_{1}^{(T)}\right]\right| 0\right\rangle\left\langle 0\left|\hat{\mathcal{W}}_{E}\left[\tilde{\mathcal{C}}_{2}^{(T)}\right]\right| 0\right\rangle}-1 .
$$

Since we are mainly interested in the large-distance behaviour of the relevant Wilson-loop correlation function, we restrict our analysis to the case of loops that do not overlap in Euclidean "time", which are characterised by

$$
b>b_{0}\left(\nu_{1}, \nu_{2}\right) \equiv r_{1}\left[f_{1}-\Theta\left(-\cos \phi_{1}\right)\right] \cos \phi_{1}-r_{2}\left[f_{2}-\Theta\left(\cos \phi_{2}\right)\right] \cos \phi_{2} \quad(\geq 0),
$$

in terms of the transverse distance and of the sizes $r_{i}$ and orientations $\phi_{i}$ of the dipoles [see eq. (2.13)]. In this case we can drop the time-ordering symbol in the numerator, obtaining

$$
\tilde{\mathcal{G}}_{E}\left(\theta ; T ; b ; \nu_{1}, \nu_{2}\right)=\frac{\left\langle 0\left|\hat{\mathcal{W}}_{E}\left[\tilde{\mathcal{C}}_{1}^{(T)}\right] \hat{\mathcal{W}}_{E}\left[\tilde{\mathcal{C}}_{2}^{(T)}\right]\right| 0\right\rangle}{\left\langle 0\left|\hat{\mathcal{W}}_{E}\left[\tilde{\mathcal{C}}_{1}^{(T)}\right]\right| 0\right\rangle\left\langle 0\left|\hat{\mathcal{W}}_{E}\left[\tilde{\mathcal{C}}_{2}^{(T)}\right]\right| 0\right\rangle}-1 .
$$

In the following we will always assume that eq. (4.7) is satisfied, unless explicitly stated, so that eq. (4.8) holds.

\subsection{Inserting a complete set of states}

The stage is now set to insert a complete set of states between the Wilson loop operators. According to the usual assumptions, such a complete set of states is made of the asymptotic (in or out) states of the theory, containing any number of the particles of the theory (including bound states) [65]. We choose in states for definiteness; the analysis is of course unchanged if one uses out states instead.

A generic asymptotic in state is characterised by its particle content, and by the momenta and the third component of the spins of the particles. We will denote by

$$
\left|\alpha,\{\vec{p}\}_{\alpha},\left\{s_{3}\right\}_{\alpha} ; i n\right\rangle
$$


a state with particle content $\alpha$, where $\alpha=\left(n_{1}, n_{2}, \ldots\right)$ is a string made up of the occupation numbers $n_{a}=n_{a}(\alpha)$ of the various particle species $a=1,2, \ldots$, characterised by their mass $m^{(a)}$ and spin $s^{(a)}$, and moreover by their baryon number, electric charge, "strangeness", "charm", "bottomness" and "topness". From now on, the latter quantum numbers will be indicated collectively as "discrete charges". Here $\{\vec{p}\}_{\alpha}=\left\{\left(p_{1}, p_{2}, p_{3}\right)\right\}_{\alpha}$ and $\left\{s_{3}\right\}_{\alpha}$ denote respectively the sets of all the momenta $\vec{p}^{(a) i}$ and all the third components of the spin $s_{3}^{(a) i}$, where the index $a=1,2, \ldots$ runs on the particle species and $i=1,2, \ldots, n_{a}(\alpha)$ on the particles of the same species. As we are interested in real-world QCD, we will consider the case $m^{(a)}>0 \forall a$; the inclusion of massless particles presents no particular difficulty, and will be briefly discussed in appendix A.1.

Let us now define the projector on the $n$-particle sector as follows:

$$
|n\rangle\left\langle n\left|\equiv \frac{1}{n !} \sum_{\alpha} \delta_{\mathcal{N}_{\alpha}, n} \mathcal{P}_{\alpha} \sum_{\left\{s_{3}\right\}_{\alpha}} \int d \Omega_{\alpha}\right| \alpha,\{\vec{p}\}_{\alpha},\left\{s_{3}\right\}_{\alpha} ; i n\right\rangle\left\langle\alpha,\{\vec{p}\}_{\alpha},\left\{s_{3}\right\}_{\alpha} ; i n\right|,
$$

where the sum is over the strings $\alpha$ with $\mathcal{N}_{\alpha} \equiv \sum_{a} n_{a}(\alpha)$ equal to $n$, the factor

$$
\mathcal{P}_{\alpha}=\frac{n !}{\prod_{a} n_{a}(\alpha) !}
$$

is due to Bose/Fermi symmetry (having factorised a $1 / n$ ! for convenience), and we have denoted

$$
\begin{aligned}
\sum_{\left\{s_{3}\right\}_{\alpha}} & =\prod_{a, n_{a}(\alpha) \neq 0} \prod_{i=1}^{n_{a}(\alpha)}\left\{\sum_{s_{3}^{(a) i}=-s^{(a)}}^{s^{(a)}}\right\} \\
\int d \Omega_{\alpha} & =\prod_{a, n_{a}(\alpha) \neq 0} \prod_{i=1}^{n_{a}(\alpha)}\left\{\int \frac{d^{3} p^{(a) i}}{(2 \pi)^{3} 2 \varepsilon^{(a) i}}\right\}, \quad \varepsilon^{(a) i}=\sqrt{\left(m^{(a)}\right)^{2}+\left(\vec{p}^{(a) i}\right)^{2}} .
\end{aligned}
$$

We are using the standard relativistic normalisation for the states. ${ }^{5}$ With this definition, the expansion of eq. (4.8) over a complete set of states reads

$$
\tilde{\mathcal{G}}_{E}\left(\theta ; T ; b ; \nu_{1}, \nu_{2}\right)=\sum_{n=1}^{\infty} \frac{\left\langle 0\left|\hat{\mathcal{W}}_{E}\left[\tilde{\mathcal{C}}_{1}^{(T)}\right]\right| n\right\rangle}{\left\langle 0\left|\hat{\mathcal{W}}_{E}\left[\tilde{\mathcal{C}}_{1}^{(T)}\right]\right| 0\right\rangle} \frac{\left\langle n\left|\hat{\mathcal{W}}_{E}\left[\tilde{\mathcal{C}}_{2}^{(T)}\right]\right| 0\right\rangle}{\left\langle 0\left|\hat{\mathcal{W}}_{E}\left[\tilde{\mathcal{C}}_{2}^{(T)}\right]\right| 0\right\rangle}=\sum_{n=1}^{\infty} \frac{1}{n !} G_{n}\left(\theta ; T ; b ; \nu_{1}, \nu_{2}\right),
$$

where we have introduced the notation

$$
\begin{aligned}
G_{n}\left(\theta ; T ; b ; \nu_{1}, \nu_{2}\right) \equiv \sum_{\alpha} \delta_{\mathcal{N}_{\alpha}, n} \mathcal{P}_{\alpha} \sum_{\left\{s_{3}\right\}_{\alpha}} \int d \Omega_{\alpha} \frac{\left\langle 0\left|\hat{\mathcal{W}}_{E}\left[\tilde{\mathcal{C}}_{1}^{(T)}\right]\right| \alpha,\{\vec{p}\}_{\alpha},\left\{s_{3}\right\}_{\alpha} ; i n\right\rangle}{\left\langle 0\left|\hat{\mathcal{W}}_{E}\left[\tilde{\mathcal{C}}_{1}^{(T)}\right]\right| 0\right\rangle} \\
\times \frac{\left\langle\alpha,\{\vec{p}\}_{\alpha},\left\{s_{3}\right\}_{\alpha} ; i n\left|\hat{\mathcal{W}}_{E}\left[\tilde{\mathcal{C}}_{2}^{(T)}\right]\right| 0\right\rangle}{\left\langle 0\left|\hat{\mathcal{W}}_{E}\left[\tilde{\mathcal{C}}_{2}^{(T)}\right]\right| 0\right\rangle}
\end{aligned}
$$

\footnotetext{
${ }^{5}$ For example, for one-particle states $\left\langle\vec{p}^{\prime}, s_{3}^{\prime} \mid \vec{p}, s_{3}\right\rangle=2 \varepsilon(2 \pi)^{3} \delta^{(3)}\left(\vec{p}^{\prime}-\vec{p}\right) \delta_{s_{3} s_{3}^{\prime}}$, for two-particle states $\left\langle\vec{p}_{1}^{\prime}, p_{2}^{\prime}, s_{31}^{\prime}, s_{32}^{\prime} \mid \vec{p}_{1}, \vec{p}_{2}, s_{31}, s_{32}\right\rangle=2 \varepsilon_{1} 2 \varepsilon_{2}(2 \pi)^{3} \delta^{(3)}\left(\vec{p}_{1}^{\prime}-\vec{p}_{1}\right)(2 \pi)^{3} \delta^{(3)}\left(\vec{p}_{2}^{\prime}-\vec{p}_{2}\right) \delta_{s_{31}^{\prime}, s_{31}}^{\prime} \delta_{s_{32}^{\prime}, s_{32}}+1 \leftrightarrow 2$, and so on.
} 
It is important to notice that, since the Wilson loop carries no flavour, contributions to this sum come only from states with vanishing "discrete charges".

We exploit now the invariance of the theory under translations along Euclidean "time" and under rotations to write

$$
\begin{aligned}
& \hat{\mathcal{W}}_{E}\left[\tilde{\mathcal{C}}_{1}^{(T)}\right]=e^{\hat{H} b} e^{-i \hat{J}_{3} \frac{\theta}{2}} \hat{\mathcal{W}}_{E}\left[\tilde{\mathcal{C}}_{0}^{(T)}\left(\nu_{1}\right)\right] e^{i \hat{J}_{3} \frac{\theta}{2}} e^{-\hat{H} b}, \\
& \hat{\mathcal{W}}_{E}\left[\tilde{\mathcal{C}}_{2}^{(T)}\right]=e^{i \hat{J}_{3} \frac{\theta}{2}} \hat{\mathcal{W}}_{E}\left[\tilde{\mathcal{C}}_{0}^{(T)}\left(\nu_{2}\right)\right] e^{-i \hat{J}_{3} \frac{\theta}{2}},
\end{aligned}
$$

where $\hat{H}$ and $\hat{J}_{3}$ are the Hamiltonian and the third component of the angular momentum, i.e., we re-express the relevant Wilson loops in terms of a rectangular loop with one (long) side parallel to the 1-axis, and one (short) side in the (3,4)-plane [recall the definition of $\tilde{\mathcal{C}}_{0}^{(T)}\left(\nu_{i}\right)$, eq. $\left.(2.14)\right]$. We can now write

$$
\begin{aligned}
G_{n}\left(\theta ; T ; b ; \nu_{1}, \nu_{2}\right)=\sum_{\alpha} & \delta_{\mathcal{N}[\alpha], n} \mathcal{P}_{\alpha} \sum_{\left\{s_{3}\right\}_{\alpha}} e^{i \theta S_{3}^{(\alpha)}\left(\left\{s_{3}\right\}_{\alpha}\right)} \int d \Omega_{\alpha} e^{-b E_{\alpha}\left(\{\vec{p}\}_{\alpha}\right)} \\
& \times W_{\alpha}^{(T)}\left(\left\{\mathcal{R}_{\frac{\theta}{2}} \vec{p}\right\}_{\alpha},\left\{s_{3}\right\}_{\alpha} ; \nu_{1}\right) \bar{W}_{\alpha}^{(T)}\left(\left\{\mathcal{R}_{-\frac{\theta}{2}} \vec{p}\right\}_{\alpha},\left\{s_{3}\right\}_{\alpha} ; \nu_{2}\right) .
\end{aligned}
$$

Here we have introduced some new notation, which we now explain. The total energy $E_{\alpha}$ and the total third component of the spin $S_{3}^{(\alpha)}$ are given by

$$
\begin{aligned}
E_{\alpha}\left(\{\vec{p}\}_{\alpha}\right) & =\sum_{a, n_{a}(\alpha) \neq 0} \sum_{i=1}^{n_{a}(\alpha)} \varepsilon^{(a) i}=\sum_{a, n_{a}(\alpha) \neq 0} \sum_{i=1}^{n_{a}(\alpha)} \sqrt{\left(m^{(a)}\right)^{2}+\left(\vec{p}^{(a) i}\right)^{2}}, \\
S_{3}^{(\alpha)}\left(\left\{s_{3}\right\}_{\alpha}\right) & =\sum_{a, n_{a}(\alpha) \neq 0} \sum_{i=1}^{n_{a}(\alpha)} s_{3}^{(a) i} .
\end{aligned}
$$

As the baryon number (and so the fermion number) must be zero for a state to contribute, the total spin $S_{3}^{(\alpha)}$ must be an integer. The Wilson-loop matrix elements are denoted by

$$
\begin{aligned}
W_{\alpha}^{(T)}\left(\{\vec{p}\}_{\alpha},\left\{s_{3}\right\}_{\alpha} ; \nu_{i}\right) & =\frac{\left\langle 0\left|\hat{\mathcal{W}}_{E}\left[\tilde{\mathcal{C}}_{0}^{(T)}\left(\nu_{i}\right)\right]\right| \alpha,\{\vec{p}\}_{\alpha},\left\{s_{3}\right\}_{\alpha} ; i n\right\rangle}{\left\langle 0\left|\hat{\mathcal{W}}_{E}\left[\tilde{\mathcal{C}}_{0}^{(T)}\left(\nu_{i}\right)\right]\right| 0\right\rangle}, \\
\bar{W}_{\alpha}^{(T)}\left(\{\vec{p}\}_{\alpha},\left\{s_{3}\right\}_{\alpha} ; \nu_{i}\right) & =\frac{\left\langle\alpha,\{\vec{p}\}_{\alpha},\left\{s_{3}\right\}_{\alpha} ; i n\left|\hat{\mathcal{W}}_{E}\left[\tilde{\mathcal{C}}_{0}^{(T)}\left(\nu_{i}\right)\right]\right| 0\right\rangle}{\left\langle 0\left|\hat{\mathcal{W}}_{E}\left[\tilde{\mathcal{C}}_{0}^{(T)}\left(\nu_{i}\right)\right]\right| 0\right\rangle} .
\end{aligned}
$$

In eq. (4.16), we have denoted the rotated three-momenta by $\left\{\mathcal{R}_{ \pm \frac{\theta}{2}} \vec{p}\right\}_{\alpha}$, where

$$
\mathcal{R}_{\varphi}=\left(\begin{array}{ccc}
\cos \varphi & \sin \varphi & 0 \\
-\sin \varphi & \cos \varphi & 0 \\
0 & 0 & 1
\end{array}\right)
$$

and it is understood that the rotation is applied to the momenta of all the particles. For our purposes, for $\theta \neq 0, \pi$, it is convenient to re-express the rotated three-momenta in terms of the variables

$$
x_{ \pm}^{(a) i}=\cos \frac{\theta}{2} p_{1}^{(a) i} \pm \sin \frac{\theta}{2} p_{2}^{(a) i} .
$$


We have

$$
\begin{aligned}
\vec{p}^{(a) i} & =\left(\frac{x_{+}^{(a) i}+x_{-}^{(a) i}}{2 \cos \frac{\theta}{2}}, \frac{x_{+}^{(a) i}-x_{-}^{(a) i}}{2 \sin \frac{\theta}{2}}, p_{3}\right), \\
\mathcal{R}_{ \pm \frac{\theta}{2}} \vec{p}^{(a) i} & =\left(x_{ \pm}^{(a) i}, \pm \cot \theta x_{ \pm}^{(a) i} \mp \frac{1}{\sin \theta} x_{\mp}^{(a) i}, p_{3}\right) .
\end{aligned}
$$

We will therefore write

$$
\begin{aligned}
W_{\alpha}^{(T)}\left(\left\{\mathcal{R}_{\frac{\theta}{2}} \vec{p}\right\}_{\alpha},\left\{s_{3}\right\}_{\alpha} ; \nu_{i}\right) & =W_{\alpha}^{(T)}\left(\left\{\left(x_{+}, \cot \theta x_{+}-\frac{1}{\sin \theta} x_{-}, p_{3}\right)\right\}_{\alpha},\left\{s_{3}\right\}_{\alpha} ; \nu_{i}\right), \\
W_{\alpha}^{(T)}\left(\left\{\mathcal{R}_{-\frac{\theta}{2}} \vec{p}\right\}_{\alpha},\left\{s_{3}\right\}_{\alpha} ; \nu_{i}\right) & =\bar{W}_{\alpha}^{(T)}\left(\left\{\left(x_{-},-\cot \theta x_{-}+\frac{1}{\sin \theta} x_{+}, p_{3}\right)\right\}_{\alpha},\left\{s_{3}\right\}_{\alpha} ; \nu_{i}\right), \\
E_{\alpha}\left(\{\vec{p}\}_{\alpha}\right) & =E_{\alpha}\left(\left\{\left(\frac{x_{+}+x_{-}}{2 \cos \frac{\theta}{2}}, \frac{x_{+}-x_{-}}{2 \sin \frac{\theta}{2}}, p_{3}\right)\right\}_{\alpha}\right)=\sum_{a, n_{a}(\alpha) \neq 0} \sum_{i=1}^{n_{a}(\alpha)} \varepsilon^{(a) i},
\end{aligned}
$$

where in terms of the new variables

$$
\varepsilon^{(a) i}=\sqrt{\left(m^{(a)}\right)^{2}+\left(\frac{x_{+}^{(a) i}+x_{-}^{(a) i}}{2 \cos \frac{\theta}{2}}\right)^{2}+\left(\frac{x_{+}^{(a) i}-x_{-}^{(a) i}}{2 \sin \frac{\theta}{2}}\right)^{2}+\left(p_{3}^{(a) i}\right)^{2}} .
$$

Furthermore, it is easy to obtain the Jacobian for the change of variables,

$$
d^{3} p^{(a) i}=\frac{1}{|\sin \theta|} d x_{+}^{(a) i} d x_{-}^{(a) i} d p_{3}^{(a) i},
$$

so that we can write

$$
\begin{aligned}
d \Omega_{\alpha} & =\frac{1}{|\sin \theta|^{\mathcal{N}_{\alpha}}} d X_{\alpha}^{+} d X_{\alpha}^{-} d \tilde{\Omega}_{\alpha} h_{\alpha}\left(\left\{\left(\frac{x_{+}+x_{-}}{2 \cos \frac{\theta}{2}}, \frac{x_{+}-x_{-}}{2 \sin \frac{\theta}{2}}, p_{3}\right)\right\}\right) \\
d X_{\alpha}^{ \pm} & =\prod_{a, n_{a}(\alpha) \neq 0} \prod_{i=1}^{n_{a}(\alpha)} \frac{d x_{ \pm}^{(a) i}}{2 \pi}, \quad d \tilde{\Omega}_{\alpha}=\prod_{a, n_{a}(\alpha) \neq 0} \prod_{i=1}^{n_{a}(\alpha)} \frac{d p_{3}^{(a) i}}{(2 \pi) 2 \tilde{\varepsilon}^{(a) i}} \\
h_{\alpha} & =\prod_{a, n_{a}(\alpha) \neq 0} \prod_{i=1}^{n_{a}(\alpha)} \frac{\tilde{\varepsilon}^{(a) i}}{\varepsilon^{(a) i}}, \quad \tilde{\varepsilon}^{(a) i}=\sqrt{\left(m^{(a)}\right)^{2}+\left(p_{3}^{(a) i}\right)^{2}}
\end{aligned}
$$

Restricting to $\theta \in(0, \pi)$, so that we can drop the absolute value from the Jacobian, we can finally write

$$
\begin{aligned}
G_{n}\left(\theta ; T ; b ; \nu_{1}, \nu_{2}\right)=\frac{1}{(\sin \theta)^{n}} \sum_{\alpha} \delta_{\mathcal{N}_{\alpha}, n} \mathcal{P}_{\alpha} \sum_{\left\{s_{3}\right\}_{\alpha}} e^{i \theta S_{3}^{(\alpha)}\left(\left\{s_{3}\right\}_{\alpha}\right)} \int d X_{\alpha}^{+} \int d X_{\alpha}^{-} \int d \tilde{\Omega}_{\alpha} \\
\times h_{\alpha}\left(\left\{\left(\frac{x_{+}+x_{-}}{2 \cos \frac{\theta}{2}}, \frac{x_{+}-x_{-}}{2 \sin \frac{\theta}{2}}, p_{3}\right)\right\}\right) e^{-b E_{\alpha}\left(\left\{\left(\frac{x_{+}+x_{-}}{2 \cos \frac{\theta}{2}}, \frac{x_{+}-x_{-}}{2 \sin \frac{\theta}{2}}, p_{3}\right)\right\}_{\alpha}\right)} \\
\times W_{\alpha}^{(T)}\left(\left\{\left(x_{+}, \cot \theta x_{+}-\frac{1}{\sin \theta} x_{-}, p_{3}\right)\right\}_{\alpha},\left\{s_{3}\right\}_{\alpha} ; \nu_{1}\right) \\
\times \bar{W}_{\alpha}^{(T)}\left(\left\{\left(x_{-},-\cot \theta x_{-}+\frac{1}{\sin \theta} x_{+}, p_{3}\right)\right\}_{\alpha},\left\{s_{3}\right\}_{\alpha} ; \nu_{2}\right) .
\end{aligned}
$$


Let us introduce one last piece of notation. Since we are interested in the limit of infinite loop length, and we expect such a limit to exist for all the matrix elements $W_{\alpha}^{(T)}, \bar{W}_{\alpha}^{(T)}$ separately, ${ }^{6}$ we define

$$
C_{n} \equiv \lim _{T \rightarrow \infty} G_{n}, \quad W_{\alpha} \equiv \lim _{T \rightarrow \infty} W_{\alpha}^{(T)}, \quad \bar{W}_{\alpha} \equiv \lim _{T \rightarrow \infty} \bar{W}_{\alpha}^{(T)}
$$

and so we write

$$
\tilde{\mathcal{C}}_{E}=\sum_{n=1}^{\infty} \frac{1}{n !} C_{n} .
$$

\subsection{Analytic continuation to Minkowski space}

The expression eq. (4.26), in the limit $T \rightarrow \infty$, is the starting point for the analytic continuation back to Minkowski space, that we now discuss. At this point we make two crucial analyticity assumptions:

1. the analytic continuation can be performed term by term, i.e., separately for the contribution of each state;

2. the matrix elements $W_{\alpha}$ and $\bar{W}_{\alpha}$, expressed in terms of the variables $x_{ \pm}^{(a) i}$, are analytic in $\theta$, in a complex domain including the real segment $(0, \pi)$ and the negative imaginary axis. $^{7}$

The first assumption is especially strong, as it requires appropriate convergence properties of the double series defined by eqs. (4.13) and (4.26) (at least in the limit $T \rightarrow \infty$ ). We will discuss later possible ways of partially relaxing this condition.

Let us now consider the various terms of eq. (4.26), in the limit $T \rightarrow \infty$, and analytically continuing $\theta$ in the complex plane, i.e., replacing $\theta \rightarrow \theta-i \chi$ with $\theta \in(0, \pi)$ and $\chi \in \mathbb{R}^{+}$. The physical, Minkowskian quantity is obtained in the limit $\theta \rightarrow 0 .{ }^{8}$ Let us start from the total energy $E_{\alpha}=\sum_{a, i} \varepsilon^{(a) i}$. We have

$$
\begin{aligned}
\varepsilon^{(a) i} & \underset{\theta \rightarrow \theta-i \chi}{\rightarrow} \sqrt{\left(m^{(a)}\right)^{2}+\left(\frac{x_{+}^{(a) i}+x_{-}^{(a) i}}{2 \cos \frac{\theta-i \chi}{2}}\right)^{2}+\left(\frac{x_{+}^{(a) i}-x_{-}^{(a) i}}{2 \sin \frac{\theta-i \chi}{2}}\right)^{2}+\left(p_{3}^{(a) i}\right)^{2}} \\
& =\sqrt{\left(m^{(a)}\right)^{2}+\left(p_{3}^{(a) i}\right)^{2}+Q^{(a) i}}=\sqrt{V^{(a) i}},
\end{aligned}
$$

\footnotetext{
${ }^{6}$ This can be understood in the LSZ framework [66, 67], where the matrix elements get replaced by the vacuum expectation values of products of appropriate interpolating fields and the Wilson loop. Due to the short-range nature of strong interactions, those parts of the loop that are too distant from the interpolating fields do not interact with them, and give contributions only to the self-interaction of the loop. As these contributions get cancelled by the normalisation factor, one expects $W_{\alpha}^{(T)}$ and $\bar{W}_{\alpha}^{(T)}$ to become almost constant beyond some "critical" loop length.

${ }^{7}$ Technically, this amounts to asking for analyticity of $W_{\alpha}$ and $\bar{W}_{\alpha}$ in the second component of the three-momenta of the particles. Notice that analyticity in all the first components of the momenta is not satisfied, as the translational invariance along direction 1 in the limit $T \rightarrow \infty$ leads to the appearence of a delta function imposing that the first component of the total momentum vanish.

${ }^{8}$ This limit gives the physical amplitude in the direct channel. The limit $\theta \rightarrow \pi$ (with negative $\chi$ ) provides the amplitude in the crossed channel, see the discussion at the end of section 2.
} 
with

$$
\begin{aligned}
Q^{(a) i}= & \left(A^{(a) i}\right)^{2}\left(\cos \frac{\theta}{2}\right)^{2}+\left(B^{(a) i}\right)^{2}\left(\sin \frac{\theta}{2}\right)^{2}+\left[\left(A^{(a) i}\right)^{2}-\left(B^{(a) i}\right)^{2}\right](\cos \theta)^{2}\left(\sinh \frac{\chi}{2}\right)^{2} \\
& +\frac{i}{2}\left[\left(B^{(a) i}\right)^{2}-\left(A^{(a) i}\right)^{2}\right] \sin \theta \sinh \chi, \\
A^{(a) i}= & \frac{1}{2} \frac{x_{+}^{(a) i}+x_{-}^{(a) i}}{\left(\cos \frac{\theta}{2} \cosh \frac{\chi}{2}\right)^{2}+\left(\sin \frac{\theta}{2} \sinh \frac{\chi}{2}\right)^{2}}, \\
B^{(a) i}= & \frac{1}{2} \frac{x_{+}^{(a) i}-x_{-}^{(a) i}}{\left(\sin \frac{\theta}{2} \cosh \frac{\chi}{2}\right)^{2}+\left(\cos \frac{\theta}{2} \sinh \frac{\chi}{2}\right)^{2}} .
\end{aligned}
$$

Convergence problems in the integration over $x_{ \pm}^{(a) i}$ and $p_{3}^{(a) i}$ may arise if there were regions with $\operatorname{Re} \varepsilon^{(a) i}<0$. This can happen only if the phase of the argument of the square root in eq. (4.29), $V^{(a) i}=\left|V^{(a) i}\right| e^{i \varphi^{(a) i}}$, grows beyond $\pm \pi$. In turn, this can happen only if $V^{(a) i}$ crosses the negative real axis, i.e., if there is a point where $\operatorname{Im} V^{(a) i}=0$ with $\operatorname{Re} V^{(a) i}<0$. However, for $\theta \in(0, \pi), \operatorname{Im} V^{(a) i}=0$ implies $\operatorname{Im} Q^{(a) i}=0$ and therefore $A^{(a) i}= \pm B^{(a) i}$, so that $\operatorname{Re} Q^{(a) i} \geq 0$ and thus $\operatorname{Re} V^{(a) i}>0$. As a consequence, as long as $\theta \neq 0, \pi$, one has $\varphi^{(a) i} \in(-\pi, \pi)$, which finally implies $\operatorname{Re} \varepsilon^{(a) i}>0 \forall a, i$, i.e., $\operatorname{Re} E_{\alpha}>0 .{ }^{9}$ On the other hand, when $\theta=0, \pi$ one has $\operatorname{Im} V^{(a) i}=0$ independently of the integration variables, while $\operatorname{Re} V^{(a) i}$ can be negative. Therefore, one should keep in mind that the limits $\theta \rightarrow 0, \pi$ can be taken only after performing the integration: a small but nonzero $\theta$ serves as a regularisation, that will be understood in the following.

Notice also that, as long as $\theta \neq 0, \pi$, one has $V^{(a) i} \neq 0$, so that no singularity appears in the quantity $h_{\alpha}$. Moreover, for $\theta=0, \pi$, these singularities are integrable, so that they cause no problem to the integration even in the limit $\theta \rightarrow 0, \pi$.

Having assumed analyticity of the matrix elements, there is no further problem in carrying out the analytic continuation to Minkowski space (i.e., in taking $\theta \rightarrow 0$ ), obtaining

$$
\begin{aligned}
C_{n}\left(-i \chi ; b ; \nu_{1}, \nu_{2}\right)= & \left(\frac{i}{\sinh \chi}\right)^{n} \sum_{\alpha} \delta_{\mathcal{N}_{\alpha}, n} \mathcal{P}_{\alpha} \sum_{\left\{s_{3}\right\}_{\alpha}} e^{\chi S_{3}^{(\alpha)}\left(\left\{s_{3}\right\}_{\alpha}\right)} \\
& \times \int d X_{\alpha}^{+} \int d X_{\alpha}^{-} \int d \tilde{\Omega}_{\alpha} h_{\alpha}\left(\left\{\left(\frac{x_{+}+x_{-}}{2 \cosh \frac{\chi}{2}}, \frac{i\left(x_{+}-x_{-}\right)}{2 \sinh \frac{\chi}{2}}, p_{3}\right)\right\}_{\alpha}\right) \\
& \times e^{-b E_{\alpha}\left(\left\{\left(\frac{x_{+}+x_{-}}{2 \cosh \frac{\chi}{2}}, \frac{i\left(x_{+}-x_{-}\right)}{2 \sinh \frac{\chi}{2}}, p_{3}\right)\right\}_{\alpha}\right)} \\
& \times W_{\alpha}\left(\left\{\left(x_{+}, i \operatorname{coth} \chi x_{+}-\frac{i}{\sinh \chi} x_{-}, p_{3}\right)\right\}_{\alpha},\left\{s_{3}\right\}_{\alpha} ; \nu_{1}\right) \\
& \times \bar{W}_{\alpha}\left(\left\{\left(x_{-},-i \operatorname{coth} \chi x_{-}+\frac{i}{\sinh \chi} x_{+}, p_{3}\right)\right\}_{\alpha},\left\{s_{3}\right\}_{\alpha} ; \nu_{2}\right)
\end{aligned}
$$

where, as we have explained above, the expression for the energy $E_{\alpha}$ is properly regularised.

\footnotetext{
${ }^{9}$ For a massless particle $m^{\left(a_{0}\right)}=0$ one has $\operatorname{Re} V^{\left(a_{0}\right) i}>0$, except at $x_{+}^{(a) i}=x_{-}^{(a) i}=p_{3}^{(a) i}=0$ where $\operatorname{Re} V^{\left(a_{0}\right) i}=0$. As a consequence, if there are massless particles in the spectrum one still has $\operatorname{Re} E_{\alpha}>0$, except on the set of states containing only massless particles of zero momentum, where $\operatorname{Re} E_{\alpha}=0$, but which is a set of zero measure.
} 
As we have explained in section 2, the Wilson-loop correlation function encodes the scattering amplitude in the high-energy regime. Therefore, physical results are obtained in the limit $\chi \rightarrow \infty$, that we now discuss. Since ${ }^{10}$

$$
\begin{gathered}
\varepsilon^{(a) i} \underset{\theta \rightarrow \theta-i \chi}{\rightarrow} \sqrt{\left(m^{(a)}\right)^{2}+\left(\frac{x_{+}^{(a) i}+x_{-}^{(a) i}}{2 \cos \frac{\theta-i \chi}{2}}\right)^{2}+\left(\frac{x_{+}^{(a) i}-x_{-}^{(a) i}}{2 \sin \frac{\theta-i \chi}{2}}\right)^{2}+\left(p_{3}^{(a) i}\right)^{2}} \\
\underset{\chi \rightarrow \infty}{\rightarrow} \sqrt{\left(m^{(a)}\right)^{2}+\left(p_{3}^{(a) i}\right)^{2}}+\mathcal{O}\left(e^{-\chi}\right)=\tilde{\varepsilon}^{(a) i}+\mathcal{O}\left(e^{-\chi}\right),
\end{gathered}
$$

we have that $h_{\alpha} \rightarrow 1$ after analytic continuation and in the large- $\chi$ limit, and that

$$
E_{\alpha}\left(\left\{\left(\frac{x_{+}+x_{-}}{2 \cosh \frac{\chi}{2}}, \frac{i\left(x_{+}-x_{-}\right)}{2 \sinh \frac{\chi}{2}}, p_{3}\right)\right\}_{\alpha}\right) \underset{\chi \rightarrow \infty}{\rightarrow} \tilde{E}_{\alpha}\left(\left\{p_{3}\right\}_{\alpha}\right)=\sum_{a, n_{a}(\alpha) \neq 0} \sum_{i=1}^{n_{a}(\alpha)} \tilde{\varepsilon}^{(a) i} .
$$

Also, to leading order, $W_{\alpha}$ is independent of $x_{-}^{(a) i}$, and $\bar{W}_{\alpha}$ is independent of $x_{+}^{(a) i}$. Finally, due to the exponential prefactor $e^{\chi S_{3}^{(\alpha)}}$, for a given particle content $\alpha$ the leading contribution comes from the spin configuration in which $s_{3}^{(a) i}=s^{(a)} \forall i \in\left\{1, \ldots, n_{a}(\alpha)\right\}$, which we will denote as $\left\{s_{3}=s\right\}_{\alpha}$. To leading order in $\chi$ we have therefore

$$
\begin{aligned}
C_{n}\left(-i \chi ; b ; \nu_{1}, \nu_{2}\right) \underset{\chi \rightarrow \infty}{\sim} & (2 i)^{n} \sum_{\alpha} \delta_{\mathcal{N}_{\alpha}, n} \mathcal{P}_{\alpha} e^{\chi\left[S_{3}^{(\alpha)}\left(\left\{s_{3}=s\right\}_{\alpha}\right)-n\right]} \int d \tilde{\Omega}_{\alpha} e^{-b \tilde{E}_{\alpha}\left(\left\{p_{3}\right\}_{\alpha}\right)} \\
& \times \mathcal{F}_{\alpha}\left(\left\{p_{3}\right\}_{\alpha}, \nu_{1}\right) \overline{\mathcal{F}}_{\alpha}\left(\left\{p_{3}\right\}_{\alpha}, \nu_{2}\right)
\end{aligned}
$$

where

$$
\begin{aligned}
\mathcal{F}_{\alpha}\left(\left\{p_{3}\right\}_{\alpha} ; \nu_{1}\right) & \equiv \int d X_{\alpha}^{+} W_{\alpha}\left(\left\{\left(x_{+}, i x_{+}, p_{3}\right)\right\}_{\alpha},\left\{s_{3}=s\right\}_{\alpha} ; \nu_{1}\right), \\
\overline{\mathcal{F}}_{\alpha}\left(\left\{p_{3}\right\}_{\alpha} ; \nu_{2}\right) & \equiv \int d X_{\alpha}^{-} \bar{W}_{\alpha}\left(\left\{\left(x_{-},-i x_{-}, p_{3}\right)\right\}_{\alpha},\left\{s_{3}=s\right\}_{\alpha} ; \nu_{2}\right),
\end{aligned}
$$

with corrections being of relative order $\mathcal{O}\left(e^{-\chi}\right) .{ }^{11}$

The result above depends crucially on our analyticity assumptions, which can however be relaxed. A possibility which is worth discussing is that the term-by-term analytic continuation can be performed only in some limited range of $\chi$ at any fixed value of $b$. Since a larger $b$ makes the coefficient of $e^{\chi\left[S_{3}^{(\alpha)}\left(\left\{s_{3}=s\right\}_{\alpha}\right)-n\right]}$ smaller, in this case we expect the range of $\chi$ to widen at larger impact parameter, including higher and higher values of the energy. Turning the argument around, we expect in this case that increasing the energy requires to go to larger impact parameter to perform the term-by-term analytic continuation. As we will discuss in the following, this could be enough for our approach to work.

\footnotetext{
${ }^{10}$ Notice that we are taking $\chi \rightarrow \infty$ before (actually, without) taking $\theta \rightarrow 0$ : we are assuming that the limit $\chi \rightarrow \infty$ commutes with the integration (for $\theta \neq 0, \pi$ ).

${ }^{11}$ Here we are assuming that $\mathcal{F}_{\alpha}$ and $\overline{\mathcal{F}}_{\alpha}$ are finite quantities, but it is of course possible that they are zero or infinite. In these cases, in eq. (4.34) they would be replaced by a finite quantity times a $\chi$-dependent suppression or enhancement factor, respectively. This would change quantitatively the result obtained with our method, but not the qualitative features of our argument.
} 


\subsection{Large- $b$ behaviour}

Before discussing the physical consequences of our result for $C_{n}$, eq. (4.34), it is useful to determine its behaviour for large impact parameter $b$. In order to do so, let us perform the change of variables

$$
p_{3}^{(a) i}=\frac{\tilde{p}_{3}^{(a) i}}{\sqrt{b m^{(a)}}} .
$$

The integration measure becomes

$$
\begin{aligned}
d \tilde{\Omega}_{\alpha} & =\prod_{a, n_{a}(\alpha) \neq 0} \prod_{i=1}^{n_{a}(\alpha)} \frac{d \tilde{p}_{3}^{(a) i}}{(2 \pi) 2 \sqrt{b m^{(a)}} \sqrt{\left(m^{(a)}\right)^{2}+\left(\frac{\tilde{p}_{3}^{(a) i}}{\sqrt{b m^{(a)}}}\right)^{2}}} \\
& =\prod_{a, n_{a}(\alpha) \neq 0} \prod_{i=1}^{n_{a}(\alpha)} \frac{1}{2 \sqrt{b m^{(a)}}} \frac{d \tilde{p}_{3}^{(a) i}}{(2 \pi) m^{(a)}}\left(1+\mathcal{O}\left(\frac{1}{b m^{(a)}}\left(\frac{\tilde{p}_{3}^{(a) i}}{m^{(a)}}\right)^{2}\right)\right),
\end{aligned}
$$

while expanding the energy $\tilde{E}_{\alpha}$ in inverse powers of $b m^{(a)}$ we obtain

$$
\tilde{E}_{\alpha}\left(\left\{\frac{\tilde{p}_{3}}{\sqrt{b m}}\right\}_{\alpha}\right)=\sum_{a, n_{a}(\alpha) \neq 0} \sum_{i=1}^{n_{a}(\alpha)}\left[m^{(a)}+\frac{1}{2 b}\left(\frac{\tilde{p}_{3}^{(a) i}}{m^{(a)}}\right)^{2}+\mathcal{O}\left(\frac{m^{(a)}}{\left(b m^{(a)}\right)^{2}}\left(\frac{\tilde{p}_{3}^{(a) i}}{m^{(a)}}\right)^{4}\right)\right] .
$$

Assuming now that $\mathcal{F}_{\alpha}\left(\{0\}_{\alpha} ; \nu_{1}\right)$ and $\overline{\mathcal{F}}_{\alpha}\left(\{0\}_{\alpha} ; \nu_{2}\right)$ are nonzero, where $\{0\}_{\alpha}$ denotes $p_{3}^{(a) i}=$ $0 \forall a, i$, and carrying out the integrations over $\tilde{p}_{3}^{(a) i}$, we obtain

$$
\begin{aligned}
C_{n}\left(-i \chi ; b ; \nu_{1}, \nu_{2}\right) \underset{\chi \rightarrow \infty, b \rightarrow \infty}{\sim} & i^{n} \sum_{\alpha} \delta_{\mathcal{N}_{\alpha}, n} \mathcal{P}_{\alpha} \mathcal{F}_{\alpha}\left(\{0\}_{\alpha} ; \nu_{1}\right) \overline{\mathcal{F}}_{\alpha}\left(\{0\}_{\alpha} ; \nu_{2}\right) \\
\times & \prod_{a}\left(\frac{1}{\sqrt{2 \pi b m^{(a)}}} e^{\chi\left[s^{(a)}-1\right]} e^{-b m^{(a)}}\right)^{n_{a}(\alpha)}
\end{aligned}
$$

with corrections being of relative order $\mathcal{O}\left(e^{-\chi}\right)$ and $\mathcal{O}\left(b^{-1}\right)$. The finiteness assumption is not crucial: if $\mathcal{F}_{\alpha}\left(\{0\}_{\alpha} ; \nu_{1}\right)$ and/or $\overline{\mathcal{F}}_{\alpha}\left(\{0\}_{\alpha} ; \nu_{2}\right)$ vanish, extra inverse powers of $b$ appear, which will not affect dramatically the high energy behaviour of the amplitude. A detailed discussion of this issue is provided in appendix A.1, where the effects due to the presence of massless particles in the spectrum are also considered.

The physical interpretation of eq. (4.39) is that the contribution to $C_{n}$ of the states $\alpha$, characterised by a given particle content, is non-negligible as long as the impact-parameter distance is smaller than or of the order of a critical "effective radius",

$$
R_{\mathrm{eff}}^{[\alpha]}(s) \equiv \frac{\sum_{a} n_{a}(\alpha)\left[s^{(a)}-1\right]}{\sum_{a} n_{a}(\alpha) m^{(a)}} \chi=\frac{\sum_{a} n_{a}(\alpha) m^{(a)} R_{\mathrm{eff}}^{(a)}(s)}{\sum_{a} n_{a}(\alpha) m^{(a)}}
$$

growing like $\sim \log s$, but with a prefactor that depends on the particle content. This means that while the ratio of effective radii corresponding to different particle contents is constant, their difference can grow logarithmically with energy. In the last passage of 
eq. (4.40) we have made explicit that the effective radius for state $\alpha$ is the weighted average of the single-particle effective radii,

$$
R_{\mathrm{eff}}^{(a)}(s) \equiv \frac{s^{(a)}-1}{m^{(a)}} \chi \equiv l_{0}^{(a)} \chi
$$

\subsection{Large- $\chi$ behaviour}

It is clear from eq. (4.39) that one cannot straightforwardly take the limit $\chi \rightarrow \infty$ of the quantity $C_{n}$. Nevertheless, since we are ultimately interested in integrating over the impact parameter $b$ to determine the elastic scattering amplitude and the total cross section, it would be enough for our purposes if we could define a variable, which is a suitable combination of $\chi$ and $b$, that encodes the energy and impact-parameter dependencies in the high-energy limit. To this extent, we define the quantity

$$
z(\chi, b) \equiv e^{c \chi} e^{-M b},
$$

where the parameters $c$ and $M$ will be determined later, and we re-express $C_{n}$ in terms of $z$ and $\chi$. Using the large- $\chi$, large- $b$ expression eq. (4.39), and including explicitly the subleading terms, we find

$$
\begin{aligned}
C_{n}\left(-i \chi ; b ; \nu_{1}, \nu_{2}\right)=i^{n} & \sum_{\alpha} \delta_{\mathcal{N}_{\alpha}, n} \mathcal{P}_{\alpha} \mathcal{F}_{\alpha}\left(\{0\}_{\alpha} ; \nu_{1}\right) \overline{\mathcal{F}}_{\alpha}\left(\{0\}_{\alpha} ; \nu_{2}\right) \\
& \times \prod_{a}\left\{\frac{e^{\chi\left[s^{(a)}-1-c \frac{m^{(a)}}{M}\right]} z^{\frac{m^{(a)}}{M}}}{\left[2 \pi \log \left(\frac{e^{c \chi}}{z}\right) \frac{m^{(a)}}{M}\right]^{\frac{1}{2}}}\right\}^{n_{a}(\alpha)} \\
& \times\left(1+\mathcal{O}\left(\frac{1}{\log \frac{e^{c \chi}}{z}}\right)+\mathcal{O}\left(e^{-\chi}\right)\right),
\end{aligned}
$$

where for clarity we have suppressed the dependence of $z$ on $\chi$ and $b$. We are considering here the case of only massive particles in the spectrum. If we now choose

$$
\frac{c}{M}=\max _{a} \frac{s^{(a)}-1}{m^{(a)}}=\max _{a} l_{0}^{(a)},
$$

assuming that it exists and that it is positive, ${ }^{12}$ we immediately see that

$$
\lim _{\chi \rightarrow \infty} e^{\chi\left[s^{(a)}-1-c \frac{m^{(a)}}{M}\right]}=\left\{\begin{array}{l}
0, \text { if } l_{0}^{(a)}<\frac{c}{M}, \\
1, \text { if } l_{0}^{(a)}=\frac{c}{M} .
\end{array}\right.
$$

Therefore, the contributions of states $\alpha$ containing particles with non-maximal effective radius, i.e., with $l_{0}^{(a)}<\frac{c}{M}$, are seen to be suppressed exponentially in $\chi$ when expressing $C_{n}$ as a function of $z$, with factors of the form $e^{-\delta \chi} z^{\beta}$ with $\delta$ and $\beta$ positive real quantities, related to the masses and spin configuration of $\alpha$.

\footnotetext{
${ }^{12}$ If the maximum in eq. (4.44) exists but is negative or zero, we can take straightforwardly $\chi \rightarrow \infty$ in eq. (4.39), obtaining either zero or a function of $b$ only. In turn, this leads to a vanishing or constant forward elastic scattering amplitude (and thus total cross section) at high energy.
} 
In principle, it is possible that there are several particles for which the ratio $l_{0}^{(a)}=\frac{s^{(a)}-1}{m^{(a)}}$ is equal to the maximum, eq. (4.44). For sure, if it is so for a particle, so it is for its antiparticle. For simplicity, we will assume that the maximum in eq. (4.44) is essentially unique, i.e., that there is a single particle-antiparticle pair that saturates it (of course, particle and antiparticle may coincide); the generalisation is straighforward, requiring only to take into account the appropriate combinatorics. If $\tilde{m}$ and $\tilde{s}$ are respectively the mass and the spin of these particles, we can conveniently choose $c=\tilde{s}-1$ and $M=\tilde{m}$. There are two possibilities.

1. Suppose that the relevant particle is a boson coinciding with its antiparticle, and therefore having vanishing discrete charges (baryon number, electric charge, "strangeness", "charm", "bottomness" and "topness"). In this case, in the limit of large $\chi$, the only terms that survive in the sum over $\alpha$ are the states $\alpha_{n}$ containing only $n$ such bosons. Since for these states $\mathcal{P}_{\alpha_{n}}=1$, eq. (4.43) simplifies to

$$
\begin{aligned}
C_{n}\left(-i \chi ; b ; \nu_{1}, \nu_{2}\right) & \underset{\chi}{\sim} \rightarrow \infty\left(\frac{i w}{\sqrt{2 \pi}}\right)^{n} \mathcal{F}_{\alpha_{n}}\left(\{0\}_{\alpha_{n}} ; \nu_{1}\right) \overline{\mathcal{F}}_{\alpha_{n}}\left(\{0\}_{\alpha_{n}} ; \nu_{2}\right) \\
& \equiv\left(\frac{i w}{\sqrt{2 \pi}}\right)^{n} C_{n}^{0}\left(\nu_{1}, \nu_{2}\right)
\end{aligned}
$$

where $w=w(\chi, z)$ is defined as

$$
w(\chi, z) \equiv z\left[\log \left(\frac{e^{(\tilde{s}-1) \chi}}{z}\right)\right]^{-\frac{1}{2}} .
$$

2. Suppose that the relevant particle is a fermion, not coinciding with its antiparticle, or a boson with nonvanishing discrete charges. ${ }^{13}$ In this case, as the selection rules on the discrete charges imply that only states with vanishing baryon number, electric charge, etc., contribute to the sum over $\alpha$, the only states that survive at large $\chi$ are those containing only pairs of the relevant particle and antiparticle. The total particle number must therefore be even, $n=2 k$, and the combinatorial factors of the relevant states $\alpha_{2 k}$ are equal to $\mathcal{P}_{\alpha_{2 k}}=\frac{(2 k) !}{(k !)^{2}}$. Therefore, eq. (4.43) simplifies to

$$
\begin{aligned}
C_{2 k}\left(-i \chi ; b ; \nu_{1}, \nu_{2}\right) & \underset{\chi}{\sim}\left(\frac{i w}{\sqrt{2 \pi}}\right)^{2 k} \frac{(2 k) !}{(k !)^{2}} \mathcal{F}_{\alpha_{2 k}}\left(\{0\}_{\alpha_{2 k}} ; \nu_{1}\right) \overline{\mathcal{F}}_{\alpha_{2 k}}\left(\{0\}_{\alpha_{2 k}} ; \nu_{2}\right) \\
& \equiv\left(\frac{i w}{\sqrt{2 \pi}}\right)^{2 k} \frac{(2 k) !}{(k !)^{2}} C_{2 k}^{0}\left(\nu_{1}, \nu_{2}\right),
\end{aligned}
$$

while the leading contribution to $C_{2 k+1}$ must contain a boson of the type discussed above in point 1 , with a nonmaximal ratio $l_{0}^{(a)}$, and therefore is exponentially suppressed in $\chi$ at fixed $w$.

\footnotetext{
${ }^{13}$ The case of a fermion coinciding with its antiparticle, and the case of a boson not coinciding with its antiparticle but having vanishing discrete charges, are not relevant to QCD.
} 
From the expressions above, it is immediate to see that $C_{n}$ depends on $\chi$ and $b$ only through the factor $w^{n}$, independently of what scenario is actually realised, ${ }^{14}$ up to subleading terms which are suppressed by at least one power of $\chi$. In conclusion, we find that

$$
\begin{aligned}
\mathcal{C}_{M}\left(\chi ; \vec{b}_{\perp} ; \nu_{1}, \nu_{2}\right) & =\tilde{\mathcal{C}}_{E}\left(\theta \rightarrow-i \chi ; b ; \nu_{1}, \nu_{2}\right) \\
\underset{\chi \rightarrow \infty}{\sim} g\left(w ; \nu_{1}, \nu_{2}\right)-1 & \equiv \begin{cases}\sum_{n=1}^{\infty} \frac{1}{n !}\left(\frac{i w}{\sqrt{2 \pi}}\right)^{n} C_{n}^{0}\left(\nu_{1}, \nu_{2}\right) & \text { (case 1) }, \\
\sum_{k=1}^{\infty} \frac{1}{(k !)^{2}}\left(\frac{i w}{\sqrt{2 \pi}}\right)^{2 k} C_{2 k}^{0}\left(\nu_{1}, \nu_{2}\right) & (\text { case } 2) .\end{cases}
\end{aligned}
$$

Here we have implicitly assumed that $\mathcal{F}_{\alpha}$ and/or $\overline{\mathcal{F}}_{\alpha}$ are nonzero at $\left\{p_{3}\right\}_{\alpha}=\{0\}_{\alpha}$ : the modifications to eq. (4.49) required when they vanish are discussed in appendix A.1. The bottom line is that at high energy, the dependence of the correlator on $\chi$ and $b$ in the "tail" region $b>b_{0}$ [see eq. (4.7)] is entirely encoded in the function $w(\chi, z(\chi, b))$ defined above in eqs. (4.42) and (4.47). Going back to our discussion of effective radii, eqs. (4.46), (4.48) and (4.49) simply state that the large- $\chi$ behaviour is determined by the particle(s) with the largest effective radius. The consequences of this fact will be explored in the next section. As a final remark, we anticipate that the important feature of the result eq. (4.49) is that in the high-energy limit the amplitude depends only on a specific combination of $\chi$ and $b$. As we will see, this allows to disentangle the energy dependence of the scattering amplitude at large $\chi$.

The validity of eq. (4.49) relies mainly on the possibility of interchanging the order in which one performs the sum over intermediate states and the analytic continuation to Minkowski space, and proving that this is actually allowed is currently out of reach in the general case. It is however possible to provide a partial justification, based on the short-range nature of strong interactions. The basic observation is that the Wilson-loop matrix elements $W_{\alpha}$ and $\bar{W}_{\alpha}$ in the limit of infinite loop length, eq. (4.18) and (4.27), can be written in factorised form to a first approximation. In the LSZ framework [66, 67], $W_{\alpha}$ and $\bar{W}_{\alpha}$ are obtained from the vacuum expectation value of the $T$-ordered product of the Wilson loop and of appropriate interpolating local fields, corresponding to each particle appearing in $\alpha$, integrated over the position of the fields. Due to the finite interaction range, in most of the configurations the interpolating fields will be far away from each other, and therefore their mutual ("particle-particle") interactions will be negligible. Furthermore, they will interact with the Wilson loop only locally (see footnote 6), so that each of them will "see" in practice a loop of infinite length and nothing else. The conclusion is that to first order one has

$$
\begin{aligned}
W_{\alpha}\left(\{\vec{p}\}_{\alpha},\left\{s_{3}\right\}_{\alpha} ; \nu_{1}\right) & \simeq \prod_{a, n_{a}(\alpha) \neq 0} \prod_{i=1}^{n_{a}(\alpha)} \lim _{T \rightarrow \infty} \frac{\left\langle 0\left|\hat{\mathcal{W}}_{E}\left[\tilde{\mathcal{C}}_{0}^{(T)}\left(\nu_{1}\right)\right]\right| \alpha, \vec{p}^{(a) i}, s_{3}^{(a) i} ; i n\right\rangle}{\left\langle 0\left|\hat{\mathcal{W}}_{E}\left[\tilde{\mathcal{C}}_{0}^{(T)}\left(\nu_{1}\right)\right]\right| 0\right\rangle} \\
& \equiv \prod_{a, n_{a}(\alpha) \neq 0} \prod_{i=1}^{n_{a}(\alpha)} W_{a}\left(\vec{p}^{(a) i}, s_{3}^{(a) i} ; \nu_{1}\right),
\end{aligned}
$$

\footnotetext{
${ }^{14}$ In case 2 , this is true only for $n=2 k$, while $C_{2 k+1}$ is exponentially suppressed in $\chi$.
} 
where $W_{a}$ are one-particle matrix elements, and similarly for $\bar{W}_{\alpha}$. From eqs. (4.13) and (4.16), and using the multinomial theorem, one finds

$$
\tilde{C}_{E} \simeq \exp \left\{\sum_{a} \sum_{s_{3}=-s^{(a)}}^{s^{(a)}} e^{i \theta s_{3}} \int d \Omega_{a} e^{-b \varepsilon^{(a)}} W_{a}\left(\mathcal{R}_{\frac{\theta}{2}} \vec{p}_{a}, s_{3} ; \nu_{1}\right) \bar{W}_{a}\left(\mathcal{R}_{-\frac{\theta}{2}} \vec{p}_{a}, s_{3} ; \nu_{2}\right)\right\}-1
$$

where $d \Omega_{a}=d^{3} p_{a} /\left[(2 \pi)^{3} 2 \varepsilon^{(a)}\right]$ is the phase-space element for a particle of type $a$, and $\varepsilon^{(a)}$ the corresponding energy. For simplicity, we have considered here only particles of the kind discussed above in point 1 , in order to avoid unnecessary complications. For particles of the kind considered above in point 2, the factorisation will be at the 2-particle level due to the selection rules, and eq. (4.51) has to be modified to include their contribution: this is discussed in appendix A.2. As the sum is now over the set of asymptotic particle species, which is finite (at least in QCD), there are no more complications due to problems of convergence of the sum, and one can safely perform the analytic continuation. Furthermore, one can explicitly verify that the resummation can be done also if one performs the analytic continuation first, and that this leads to the same result. This proves that the term-byterm analytic continuation is justified when particle-particle interactions can be neglected. Including the corrections due to particle-particle interactions will modify the expression above, but we think that it is reasonable to assume that it will not spoil the possibility of interchanging summation and analytic continuation.

It is worth noting that even if the term-by-term analytic continuation can be performed only for a limited range of $\chi$ at any fixed $b$, which we expect to include higher and higher energies as we increase the impact parameter, eq. (4.43) shows that in this case we could nevertheless take the large- $\chi$ limit at fixed $w$, which amounts to take at the same time the large- $\chi$ and the large- $b$ limit. This means that in this case eq. (4.49) would define the coefficients of a convergent power series at least in some limited range of $w$. In this case, even though the power series representation would be valid only within its finite radius of convergence, it is possible that the analytic function obtained by resumming the series could be analytically continued (in $w$ ) outside the radius of convergence.

More precisely, one can formulate the following condition. Let us assume that the double series defined by eqs. (4.13) and (4.26), re-expressed in terms of the complex variable ${ }^{15}$

$$
w=\frac{e^{i \theta(\tilde{s}-1)} e^{-b \tilde{m}}}{\sqrt{b \tilde{m}}}
$$

and of $\theta$, is such that a term-by-term analytic continuation of $\theta \rightarrow-i \chi$ at fixed $w$ can be performed, for $w \in \mathcal{D}_{0}$ with $\mathcal{D}_{0}$ some complex domain. Possibly, the analytic continuation has to be understood as $\theta \rightarrow \epsilon-i \chi$, followed by the limit $\epsilon \rightarrow 0$. Let us assume furthermore that in a subdomain $\mathcal{D}_{1} \subseteq \mathcal{D}_{0}$, that we assume to contain part of the positive real axis in the complex- $w$ plane, at least as a boundary, it is possible to take $\chi \rightarrow \infty$ at fixed $w$. Taking $w \in \mathcal{D}_{1}$, performing the analytic continuation in $\theta$, and setting

$$
\mathcal{C}_{M}\left(\chi ; \vec{b}_{\perp} ; \nu_{1}, \nu_{2}\right)=\tilde{\mathcal{C}}_{E}\left(-i \chi ; b ; \nu_{1}, \nu_{2}\right)=F\left(w(\chi, z(b, \chi)) ; \chi ; \nu_{1}, \nu_{2}\right),
$$

\footnotetext{
${ }^{15}$ Here we are assuming $\tilde{s}>1$.
} 
according to the discussion above one has that $F$ can be written as follows,

$$
F\left(w ; \chi ; \nu_{1}, \nu_{2}\right)=g\left(w ; \nu_{1}, \nu_{2}\right)+g_{1}\left(w ; \chi ; \nu_{1}, \nu_{2}\right),
$$

with $g_{1} \rightarrow 0$ as $\chi \rightarrow \infty$ at fixed $w$. Moreover, if $F$ and $g$ can be analytically extended beyond $\mathcal{D}_{1}$ including a larger part (possibly all) of the real axis, then so can be $g_{1}$, which will vanish as $\chi \rightarrow \infty$ in the whole extended domain.

\section{$5 \quad$ Elastic scattering amplitude and total cross section}

We are now in a position to discuss the high-energy behaviour of the meson-meson elastic scattering amplitude and of the corresponding total cross section, so completing the argument outlined in section 3. For this purpose, it is useful to analyse first the consequences of unitarity on the relevant Wilson loop correlators.

It is clear from eqs. (2.1) and (2.6), and from the definition of the scattering amplitude in the impact-parameter representation,

$$
\mathcal{M}_{(h h)}(s, t)=-2 i s \int d^{2} \vec{z}_{\perp} e^{i \vec{q}_{\perp} \cdot \vec{z}_{\perp}} a_{(h h)}\left(s, \vec{z}_{\perp}\right),
$$

that $a_{(h h)}$ coincides with the Minkowskian Wilson-loop correlator averaged over the dipole variables, i.e.,

$$
a_{(h h)}\left(s, \vec{z}_{\perp}\right)=\left\langle\left\langle\mathcal{C}_{M}\left(\chi ; \vec{z}_{\perp} ; \nu_{1}, \nu_{2}\right)\right\rangle\right\rangle .
$$

It is well known [68-70] that the impact-parameter amplitude satisfies the unitarity constraint $\left|a_{(h h)}\left(s, \vec{z}_{\perp}\right)+1\right| \leq 1, \forall \vec{z}_{\perp}$. Therefore, if the description of the scattering process in terms of dipoles, that we are using in this work, is to lead to physically meaningful results, then the normalised Wilson-loop correlator in Minkowski space has to satisfy the following unitarity constraint (at least in the large- $\chi$ limit),

$$
\left|\left\langle\left\langle\mathcal{C}_{M}\left(\chi ; \vec{z}_{\perp} ; \nu_{1}, \nu_{2}\right)\right\rangle\right\rangle+1\right| \leq 1, \quad \forall \vec{z}_{\perp}
$$

If the dipole picture is correctly describing soft high-energy processes, and since the constraint eq. (5.3) has to be satisfied for all the species of colliding mesons, i.e., for all the physical choices of the wave functions $\psi_{1,2}$ in eq. (2.1), we expect a stronger unitarity constraint to be satisfied, namely

$$
\left|\mathcal{C}_{M}\left(\chi ; \vec{z}_{\perp} ; \nu_{1}, \nu_{2}\right)+1\right| \leq 1, \quad \forall \vec{z}_{\perp}, \nu_{1}, \nu_{2} .
$$

In particular, choosing $\vec{z}_{\perp}=\vec{b}_{\perp}=(b, 0)$ parallel to the 2-axis, we have

$$
\left|\mathcal{C}_{M}\left(\chi ; \vec{b}_{\perp} ; \nu_{1}, \nu_{2}\right)+1\right| \leq 1, \quad \forall b, \nu_{1}, \nu_{2} .
$$

For $b>b_{0}$, where the analysis of the previous section applies, we have that $\mathcal{C}_{M}\left(\chi ; \vec{b}_{\perp}\right)+1=$ $g(w(\chi, z(\chi, b)))+g_{1}(w(\chi, z(\chi, b)) ; \chi)$, with $g_{1} \rightarrow 0$ as $\chi \rightarrow \infty$ with $w$ fixed [see eq. (4.49)]. Here we have dropped all the irrelevant dependencies. The function $g$ is just the highenergy, large- $b$ approximation of the normalised Wilson-loop correlator, expressed as a 
function of $w$. As already remarked, one can keep $w$ fixed to any non-negative real value as $\chi \rightarrow \infty$ if one also properly takes $b \rightarrow \infty$. We have therefore, according to eq. (5.5),

$$
\left|g\left(w ; \nu_{1}, \nu_{2}\right)\right|=\lim _{\substack{\chi, b \rightarrow \infty \\ w \text { fixed }}}\left|\mathcal{C}_{M}\left(\chi ; \vec{b}_{\perp} ; \nu_{1}, \nu_{2}\right)+1\right| \leq 1 \quad \forall w \geq 0, \nu_{1}, \nu_{2},
$$

i.e., $g$ is a bounded function.

\subsection{Asymptotic behaviour of the total cross section}

Recalling eq. (2.8), and exploiting the optical theorem, we obtain for the total cross section

$$
\sigma_{\text {tot }}^{(h h)}(s) \underset{s \rightarrow \infty}{\sim} \frac{1}{s} \operatorname{Im} \mathcal{M}_{(h h)}(s, t=0)=-4 \pi \operatorname{Re}\left\langle\left\langle\int_{0}^{\infty} d b b \mathcal{C}_{M}\left(\chi ; \vec{b}_{\perp} ; \nu_{1}, \nu_{2}\right)\right\rangle_{\varphi},\right.
$$

where $\vec{b}_{\perp}=(b, 0)$ is parallel to the 2-axis, $\chi \simeq \log \left(s / m^{2}\right)$ at high energy, and $\left\langle\langle\ldots\rangle_{\varphi}\right.$ has been defined in eq. (2.8). The integral in eq. (5.7) is conveniently split into two parts,

$$
\begin{aligned}
\int_{0}^{\infty} d b b \mathcal{C}_{M}\left(\chi ; \vec{b}_{\perp} ; \nu_{1}, \nu_{2}\right)= & \int_{0}^{b_{0}\left(\nu_{1}, \nu_{2}\right)} d b b \mathcal{C}_{M}\left(\chi ; \vec{b}_{\perp} ; \nu_{1}, \nu_{2}\right) \\
& +\int_{b_{0}\left(\nu_{1}, \nu_{2}\right)}^{\infty} d b b \mathcal{C}_{M}\left(\chi ; \vec{b}_{\perp} ; \nu_{1}, \nu_{2}\right)
\end{aligned}
$$

where $b_{0}$ has been defined in eq. (4.7). We expect from unitarity [see eq. (5.5)] that the first term is bounded by a $\chi$-independent function, and so we will focus on the second term. Here we can use the approximate expression for $\mathcal{C}_{M}$ obtained in the previous section, eq. (4.49). Changing variables to $z$, as defined in the previous section, i.e.,

$$
z=e^{(\tilde{s}-1) \chi} e^{-\tilde{m} b}, \quad \tilde{m} b=\log \frac{e^{(\tilde{s}-1) \chi}}{z}, \quad \tilde{m} d b=-\frac{d z}{z},
$$

and setting $e^{-b_{0} \tilde{m}}=\Lambda$, with $\Lambda=\Lambda\left(\nu_{1}, \nu_{2}\right)$, we can write

$$
\begin{aligned}
J\left(\chi ; \nu_{1}, \nu_{2}\right) \equiv- & \int_{b_{0}\left(\nu_{1}, \nu_{2}\right)}^{\infty} d b b \mathcal{C}_{M}\left(\chi ; \vec{b}_{\perp} ; \nu_{1}, \nu_{2}\right) \\
& \underset{\chi \rightarrow \infty}{\sim} \frac{1}{\tilde{m}^{2}} \int_{0}^{e^{(\tilde{s}-1) \chi} \Lambda} \frac{d z}{z} \log \left(\frac{e^{(\tilde{s}-1) \chi}}{z}\right)\left[1-g\left(w(\chi, z) ; \nu_{1}, \nu_{2}\right)\right],
\end{aligned}
$$

where $w(\chi, z)$ has been defined in eq. (4.47), and $g$ is defined in eq. (4.49). One could in principle use $w$ itself as integration variable, but for our purposes it is more convenient to follow a different strategy. Let $z=\xi(\chi) z^{\prime}$, and let us require that $\xi$ is such that

$$
w(\chi, z)=w\left(\chi, \xi(\chi) z^{\prime}\right)=\frac{\xi(\chi)}{\sqrt{\log \left(\frac{e^{(\tilde{s}-1) \chi}}{\xi(\chi)}\right)}} \frac{z^{\prime}}{\sqrt{1+\frac{\log \left(\frac{1}{z^{\prime}}\right)}{\log \left(\frac{e^{(\tilde{s}-1) \chi}}{\xi(\chi)}\right)}}} \equiv \frac{z^{\prime}}{\sqrt{1+\frac{\log \left(\frac{1}{z^{\prime}}\right)}{\log \left(\frac{e^{(\tilde{s}-1) \chi}}{\xi(\chi)}\right)}}} .
$$

The reason to do this is that now eq. (5.10) depends on $\chi$ only through the variable $\eta$,

$$
e^{\eta} \equiv \frac{e^{(\tilde{s}-1) \chi}}{\xi(\chi)}, \quad \xi^{2}+\log \xi=(\tilde{s}-1) \chi, \quad \eta=(\tilde{s}-1) \chi-\log \xi=\xi^{2} .
$$


The equation for $\xi$ in eq. (5.12) can be solved, yielding

$$
\eta=\xi^{2}=\frac{1}{2} W\left(2 e^{2(\tilde{s}-1) \chi}\right)
$$

where $W(z)$ is the Lambert $W$ function [73], defined by the equation

$$
z=W(z) e^{W(z)} .
$$

For large positive $z, W(z)=\log z-\log \log z+\frac{\log \log z}{\log z}+\ldots$, and so we obtain at large energy

$$
\eta=(\tilde{s}-1) \chi-\frac{1}{2} \log [(\tilde{s}-1) \chi]+\frac{\log [(\tilde{s}-1) \chi]}{4(\tilde{s}-1) \chi}+\ldots
$$

Dropping now the prime, rescaling $z \rightarrow \Lambda z$, and setting for notational convenience

$$
\tilde{g}\left(z ; \nu_{1}, \nu_{2}\right)=g\left(z \Lambda\left(\nu_{1}, \nu_{2}\right) ; \nu_{1}, \nu_{2}\right)
$$

we can recast eq. (5.10) in the compact form

$$
J\left(\chi ; \nu_{1}, \nu_{2}\right) \underset{\chi \rightarrow \infty}{\sim} \frac{1}{\tilde{m}^{2}} \int_{0}^{e^{\eta}} \frac{d z}{z} \log \left(\frac{e^{\eta}}{\Lambda z}\right)\left(1-\tilde{g}\left(z ; \nu_{1}, \nu_{2}\right)\right),
$$

where we have neglected terms of order $\mathcal{O}\left(\eta^{-1}\right)$ appearing in eq. (5.10) [see eq. (5.11)]. Clearly, the constraint eq. (5.6) holds also for $\tilde{g}$.

In order to determine the high-energy behaviour of $J$, it is convenient to split it into three parts, $J=J_{1}-J_{2}+J_{3}$, with

$$
\begin{aligned}
& J_{1}\left(\chi ; \nu_{1}, \nu_{2}\right)=\frac{1}{\tilde{m}^{2}} \int_{1}^{e^{\eta}} \frac{d z}{z} \log \left(\frac{e^{\eta}}{\Lambda z}\right), \\
& J_{2}\left(\chi ; \nu_{1}, \nu_{2}\right)=\frac{1}{\tilde{m}^{2}} \int_{1}^{e^{\eta}} \frac{d z}{z} \log \left(\frac{e^{\eta}}{\Lambda z}\right) \tilde{g}\left(z ; \nu_{1}, \nu_{2}\right), \\
& J_{3}\left(\chi ; \nu_{1}, \nu_{2}\right)=\frac{1}{\tilde{m}^{2}} \int_{0}^{1} \frac{d z}{z} \log \left(\frac{e^{\eta}}{\Lambda z}\right)\left(1-\tilde{g}\left(z ; \nu_{1}, \nu_{2}\right)\right) .
\end{aligned}
$$

The first integral can be easily computed, and gives

$$
J_{1}\left(\chi ; \nu_{1}, \nu_{2}\right)=\frac{1}{\tilde{m}^{2}}\left[\frac{1}{2} \eta^{2}+\eta \log \frac{1}{\Lambda}\right] .
$$

Moreover, the dependence of $J_{3}$ on $\eta$ is easily exposed,

$$
J_{3}\left(\chi ; \nu_{1}, \nu_{2}\right)=\frac{1}{\tilde{m}^{2}}\left[\eta c_{1}\left(\nu_{1}, \nu_{2}\right)+c_{2}\left(\nu_{1}, \nu_{2}\right)\right],
$$

where $c_{1,2}$ are functions of the dipole variables only, so that $J_{3}$ is subleading in $\eta$. Finally, using the unitarity constraint eq. (5.5), or eq. (5.6), we can bound $J_{2}$,

$$
\left|J_{2}\left(\chi ; \nu_{1}, \nu_{2}\right)\right| \leq J_{1}\left(\chi ; \nu_{1}, \nu_{2}\right)
$$


which leads to the following bound on the total cross section at high energy,

$$
\sigma_{\text {tot }}^{(h h)}(s) \underset{s \rightarrow \infty}{\simeq} 4 \pi \operatorname{Re}\left\langle\left\langle J\left(\chi ; \nu_{1}, \nu_{2}\right)\right\rangle_{\varphi} \underset{s \rightarrow \infty}{\lesssim} 4 \pi \frac{\eta^{2}}{\tilde{m}^{2}} \simeq 4 \pi \frac{(\tilde{s}-1)^{2}}{\tilde{m}^{2}}\left(\log \frac{s}{m^{2}}\right)^{2},\right.
$$

where we have used eq. (5.15) and the fact that $\left\langle\langle 1\rangle_{\varphi}=1\right.$. Notice that the subleading terms neglected in the last passage are of order $\mathcal{O}(\log s \cdot \log \log s)$, as can be seen again from eq. (5.15). This "Froissart-like" bound is a consequence of the analyticity and unitarity properties assumed for the Wilson-loop correlator, and of the existence of a maximal (and positive) ratio $\frac{(\tilde{s}-1)}{\tilde{m}}$ in the hadronic spectrum. The origin of the prefactor is therefore rather different than in the original derivation of the FLM bound, where it is related to the position of the lowest singularity in the $t$-channel.

\subsection{Universality of total cross sections}

From eqs. (5.19) and (5.21), we can write for the asymptotic energy dependence of the total cross section

$$
\sigma_{\text {tot }}^{(h h)}(s) \underset{s \rightarrow \infty}{\sim} 2 \pi \frac{(\tilde{s}-1)^{2}}{\tilde{m}^{2}}\left[1-\left\langle\left\langle\operatorname{Re} \Delta\left(\nu_{1}, \nu_{2}\right)\right\rangle\right\rangle_{\varphi}\right]\left(\log \frac{s}{m^{2}}\right)^{2},
$$

where we have set $J_{2} \sim \frac{1}{2} \Delta\left(\nu_{1}, \nu_{2}\right) \eta^{2}$ at high energy, compatibly with eq. (5.21). Since the quantity $\Delta$ can in principle depend nontrivially on the dipole variables, in general the resulting total cross section will be nonuniversal. In order to have universality, either $\Delta$ is purely imaginary (or even zero), or $\operatorname{Re} \Delta$ is independent of $\nu_{1,2}$, or for some reason the average of $\operatorname{Re} \Delta$ over the dipole variables is universal, which would still be rather natural if only the average over the orientations of the dipoles and over the momentum fractions were required.

To investigate the issue of universality, we exploit eq. (5.6) to write

$$
\tilde{g}\left(z ; \nu_{1}, \nu_{2}\right)=e^{-\rho\left(z ; \nu_{1}, \nu_{2}\right)+i \phi\left(z ; \nu_{1}, \nu_{2}\right)},
$$

with $\rho, \phi \in \mathbb{R}$ and $\rho \geq 0$. Let us now consider several interesting cases.

1. The simplest possibility is that $\rho(z \rightarrow \infty) \rightarrow \infty$, in which case $\tilde{g}(z \rightarrow \infty) \rightarrow 0$, and we see that in eq. (5.18) we can push the upper limit of integration in $J_{2}$ to infinity, obtaining a finite quantity. ${ }^{16}$ This implies that

$$
\begin{aligned}
J_{2}\left(\chi ; \nu_{1}, \nu_{2}\right)= & \frac{\eta}{\tilde{m}^{2}}\left[\int_{1}^{\infty} \frac{d z}{z} \tilde{g}\left(z ; \nu_{1}, \nu_{2}\right)+o(1)\right] \\
& +\frac{1}{\tilde{m}^{2}} \int_{1}^{\infty} \frac{d z}{z} \log \left(\frac{1}{\Lambda z}\right) \tilde{g}\left(z ; \nu_{1}, \nu_{2}\right)+o(1)
\end{aligned}
$$

i.e., $J_{2}=\mathcal{O}(\eta)$ and therefore $\Delta=0$, so that universality is obtained. In physical terms, this case corresponds to the Minkowskian Wilson-loop correlator vanishing at large $\chi$ for any fixed $b$ (at least for any fixed $b>b_{0}$ ), so that in this limit the impact-parameter amplitude goes to 1 . In other words, this would directly correspond to the usual assumption of particles behaving in a scattering process as black disks with energy-dependent radius.

\footnotetext{
${ }^{16}$ This requires $\tilde{g}$ to vanish at least as fast as $|\log z|^{-2-\epsilon}$.
} 
2. Another possibility we want to discuss is that of an amplitude that keeps oscillating as $z \rightarrow \infty$, i.e., $\phi(z) \sim \phi_{0} z^{\lambda}, \lambda>0$, as $z \rightarrow \infty$. Separating the leading contribution from the rest, setting $\tilde{g}(z)=e^{-\rho(z)} e^{i\left(\phi(z)-\phi_{0} z^{\lambda}\right)} e^{i \phi_{0} z^{\lambda}}=\tilde{A}(z) e^{i \phi_{0} z^{\lambda}}$, and changing variables to $y=z^{\lambda}$ in the integral for $J_{2}$, eq. (5.18), we obtain

$$
J_{2}\left(\chi ; \nu_{1}, \nu_{2}\right)=\frac{1}{\lambda^{2} \tilde{m}^{2}} \int_{1}^{e^{\lambda \eta}} \frac{d y}{y} \log \left(\frac{e^{\lambda \eta}}{\Lambda^{\lambda} y}\right) e^{i \phi_{0} y} \tilde{A}\left(y^{\frac{1}{\lambda}}\right) .
$$

Since the function $f_{\lambda}(y)=\frac{1}{y} \log \left(\frac{e^{\lambda \eta}}{\Lambda^{\lambda} y}\right) \theta(y-1)$ is $\mathcal{L}^{2}(\mathbb{R})$ and $\tilde{A}$ is bounded, we can push the upper limit of integration to infinity, obtaining the Fourier transform of $f_{\lambda} \tilde{A}$ at $\phi_{0}$. As a consequence, the leading behaviour of $J_{2}$ is only $\mathcal{O}(\eta)$; therefore $\Delta=0$, and the colliding particles behave effectively as black disks. Interestingly, we recover the same result obtained in the previous case starting from completely different assumptions. The key point is the possibility of pushing the upper limit of integration to infinity in $J_{2}$, so that eq. (5.25) above holds: any time that this can be done, no matter under what conditions, we will get $\Delta=0$ and universality of the total cross sections.

3. The last case we want to discuss is that in which both $\phi$ and $\rho$ become independent of $z$ at large $z$ while remaining finite, i.e., $\phi(z \rightarrow \infty)=\phi_{\infty}\left(\nu_{1}, \nu_{2}\right)$, and $\rho(z \rightarrow \infty) \rightarrow \rho_{\infty}\left(\nu_{1}, \nu_{2}\right)$. In this case, we can write

$$
J_{2}\left(\chi ; \nu_{1}, \nu_{2}\right)=\frac{1}{\tilde{m}^{2}} \int_{1}^{e^{\eta}} \frac{d z}{z} \log \left(\frac{e^{\eta}}{\Lambda z}\right)\left[e^{-\rho_{\infty}\left(\nu_{1}, \nu_{2}\right)} e^{i \phi_{\infty}\left(\nu_{1}, \nu_{2}\right)}+r\left(z ; \nu_{1}, \nu_{2}\right)\right],
$$

where the quantity $r\left(z ; \nu_{1}, \nu_{2}\right) \equiv e^{-\rho\left(z ; \nu_{1}, \nu_{2}\right)} e^{i \phi\left(z ; \nu_{1}, \nu_{2}\right)}-e^{-\rho_{\infty}\left(\nu_{1}, \nu_{2}\right)} e^{i \phi_{\infty}\left(\nu_{1}, \nu_{2}\right)}$ vanishes as $z \rightarrow \infty$. As a consequence, in the corresponding contribution to the integral one can again push the upper limit of integration to infinity, obtaining a finite quantity. ${ }^{17}$ In turn, this implies that this contribution grows at most like $\eta$ at large energy. A leading contribution to $J_{2}$, proportional to $\chi^{2}$, is however possible, and reads

$$
J_{2}\left(\chi ; \nu_{1}, \nu_{2}\right)=\frac{e^{-\rho_{\infty}\left(\nu_{1}, \nu_{2}\right)}}{2 \tilde{m}^{2}} e^{i \phi_{\infty}\left(\nu_{1}, \nu_{2}\right)} \eta^{2}+\mathcal{O}(\eta) .
$$

The leading contribution to the forward scattering amplitude is therefore

$$
\mathcal{M}_{(h h)}(s, 0)=i s \frac{2 \pi(\tilde{s}-1)^{2}}{\tilde{m}^{2}} \chi^{2}\left(1-e^{-\kappa^{(h h)}} e^{i \varphi^{(h h)}}\right)
$$

where we have set

$$
e^{-\kappa^{(h h)}} e^{i \varphi^{(h h)}}=\left\langle\left\langle e^{-\rho_{\infty}\left(\nu_{1}, \nu_{2}\right)} e^{i \phi_{\infty}\left(\nu_{1}, \nu_{2}\right)}\right\rangle_{\varphi},\right.
$$

with $\kappa^{(h h)}, \varphi^{(h h)} \in \mathbb{R}$ and $\kappa^{(h h)} \geq 0$. In general, both $\kappa^{(h h)}$ and $\varphi^{(h h)}$ will depend on the kind of particles involved in the scattering process. However, not any value of $\varphi^{(h h)}$ is allowed, due to the constraint on the phase of scattering amplitudes at high energy coming from analyticity and crossing symmetry (see, e.g., ref. [71]). Due to this constraint, the crossing-symmetric part of the amplitude must be purely imaginary at high energy.

\footnotetext{
${ }^{17}$ This requires $r$ to vanish at least as fast as $|\log z|^{-2-\epsilon}$.
} 
If, for simplicity, the hadronic wave functions are chosen to be invariant under rotations and under the exchange $f_{i} \rightarrow 1-f_{i}$, as mentioned in section 2 , the scattering amplitude is automatically crossing-symmetric, and therefore one must have $\varphi^{(h h)}=0, \pi$. Since this must hold for all physical choices of the hadronic wave functions, the simplest way to achieve this is to have $\phi_{\infty}\left(\nu_{1}, \nu_{2}\right) \equiv 0$, or $\pi$, independently of $\nu_{i}$. This would "naturally" lead to universality of total cross sections only if at the same time $\rho_{\infty}\left(\nu_{1}, \nu_{2}\right)=\bar{\rho}_{\infty}$, independently of $\nu_{i}$ :

$$
\sigma_{\text {tot }}^{(h h)}(s) \underset{s \rightarrow \infty}{\sim} 2 \pi \frac{(\tilde{s}-1)^{2}}{\tilde{m}^{2}}\left[1 \mp e^{-\bar{\rho}_{\infty}}\right]\left(\log \frac{s}{m^{2}}\right)^{2}
$$

where the upper (lower) sign corresponds to $\phi_{\infty}\left(\nu_{1}, \nu_{2}\right)=0(\pi)$. According to the sign, one would have in this case either grey-disk $\left(\phi_{\infty}=0\right)$ or anti-shadowing $[74,75]\left(\phi_{\infty}=\pi\right)$ behaviour. In particular, if $\bar{\rho}_{\infty}=0$ one would have respectively no $\log ^{2} s$ contribution, or saturation of the unitarity limit. Although we cannot exclude that this scenario is realised, we find it rather unpleasing, since it requires several extra conditions to be met in order to achieve universality, and contains one parameter (i.e., $1 \mp e^{-\bar{\rho}_{\infty}}$ ) that remains undetermined at this stage. In the remaining part of this section we will discuss only the first two cases, with $\Delta=0$.

If eq. (5.25) holds, we can set for convenience

$$
\int_{1}^{\infty} \frac{d z}{z} \tilde{g}\left(z ; \nu_{1}, \nu_{2}\right)=-C\left(\nu_{1}, \nu_{2}\right)+c_{1}\left(\nu_{1}, \nu_{2}\right)+\log \frac{1}{\Lambda}
$$

and write for $J$

$$
4 \pi J\left(\chi ; \nu_{1}, \nu_{2}\right) \underset{s \rightarrow \infty}{\sim} \frac{2 \pi}{\tilde{m}^{2}} \eta^{2}+\frac{4 \pi}{\tilde{m}^{2}}\left\langle\left\langle C\left(\nu_{1}, \nu_{2}\right)\right\rangle\right\rangle_{\varphi} \eta,
$$

where $\eta$ has been defined in eq. (5.13). From this we find that the total cross section behaves asymptotically as

$$
\sigma_{\text {tot }}^{(h h)}(s) \underset{s \rightarrow \infty}{\sim} 2 \pi \frac{(\tilde{s}-1)^{2}}{\tilde{m}^{2}}\left(\log \frac{s}{m^{2}}\right)^{2}-2 \pi \frac{(\tilde{s}-1)}{\tilde{m}^{2}} \log \frac{s}{m^{2}} \cdot \log \log \frac{s}{m^{2}}+Q^{(h h)} \log \frac{s}{m^{2}},
$$

where we have used the large-s behaviour of $\eta$ [see eq. (5.15)], we have introduced the (process-dependent) coefficient $Q^{(h h)}$ of the $\log s$ term, and discarded further subleading terms. There are three important consequences of this expression.

1. The leading energy dependence is of the form $\sigma_{\text {tot }}^{(h h)}(s) \sim B \log ^{2} s$ for $s \rightarrow \infty$, with universal $B$, i.e., independent of the colliding mesons. One easily sees that extending the calculation to the case of mesons with different masses this term remains unaffected.

2. The universal coefficient $B=2 \pi \frac{(\tilde{s}-1)^{2}}{\tilde{m}^{2}}$ can be entirely determined from the hadronic spectrum.

3. The first subleading correction in energy is proportional to $\log s \cdot \log \log s$, and it is also universal.

All of the above remains true if extra powers of $b$ have to be taken into account in the large- $b$ behaviour, as mentioned after eq. (4.39). More precisely, the coefficient of the 
subleading $\log s \cdot \log \log s$ term gets an extra (universal) factor, while the leading term remains unaltered. A detailed discussion is reported in appendix A.1, where we also make contact with the parameterisations discussed in ref. [59].

Let us now briefly discuss the energy dependence of further subleading corrections. The $o(1)$ terms in the first square bracket in eq. (5.25) are expected to vanish quite fast, and if they vanish at least as fast as $\eta^{-1}$, then they simply give a constant contribution to the total cross section, plus vanishing terms. The terms that have been neglected in the integrand passing from eq. (5.10) to eq. (5.17) are of order $\mathcal{O}\left(\eta^{-1}\right)$, and so can provide at most a contribution of order $\mathcal{O}(\eta)$ to the total cross section. However, a direct calculation shows that in the cases 1-3 discussed above they only contribute to $\mathcal{O}(1)$. The terms that have been discarded from eq. (4.49) on are bounded [this follows from eqs. (5.5) and (5.6)] and suppressed by a factor $\left[\log \left(\frac{e^{\eta}}{z}\right)\right]^{-1}$, so they can yield at most a contribution of order $\mathcal{O}(\eta)$ to the total cross section, which has to be included in $Q^{(h h)}$ in eq. (5.34). In any case, eq. (5.34) above gives the leading contribution to the total cross section, in the case $\operatorname{Re} \Delta=0$.

\subsection{Elastic scattering amplitude at high energy}

To conclude this section, we want to briefly discuss what happens to the elastic scattering amplitude if the conditions leading to $\Delta=0$ are met. As these conditions correspond in practice to particles behaving as black disks at high energy, one expects that the usual results obtained in the black-disk model hold. For simplicity, we will work with rotationinvariant wave functions, as appropriate for example for unpolarised scattering processes. In this case, recalling eq. (2.9), the elastic scattering amplitude reads

$$
\mathcal{M}_{(h h)}(s, t)=-4 \pi i s\left\langle\left\langle\int_{0}^{\infty} d b b J_{0}(b \sqrt{-t}) \mathcal{C}_{M}\left(\chi \simeq \log \left(s / m^{2}\right) ; \vec{b}_{\perp} ; \nu_{1}, \nu_{2}\right)\right\rangle_{0}\right.
$$

If $\tilde{g}(z)$ defined in eq. (5.16) vanishes sufficiently fast at large $z$ (any power law will do), or if it oscillates wildly, one can repeat the argument carried out above for the total cross section, taking now into account the Bessel function $J_{0}(b \sqrt{-t})$ appearing in eq. (5.35). A simple calculation then shows that to leading order

$$
\mathcal{M}_{(h h)}(s, t) \underset{s \rightarrow \infty}{\sim} 4 \pi i s\left(\frac{\eta}{\tilde{m}}\right)^{2}\left(\frac{\tilde{m}}{q \eta}\right) J_{1}\left(\frac{q \eta}{\tilde{m}}\right)=4 \pi i s\left(\frac{\eta}{\tilde{m}}\right)^{2} \frac{J_{1}(\varrho)}{\varrho},
$$

where we have set $\varrho=\varrho(\chi, q) \equiv \frac{q \eta}{\tilde{m}}$. This is actually a black-disk scattering amplitude with radius $\frac{\eta}{\tilde{m}}$. A few remarks are in order.

1. The amplitude is purely imaginary at high energy, as expected from analyticity and crossing symmetry, and from the fact that it is $\mathcal{M}_{(h h)}(s, t) \propto s$ up to logarithms (see, e.g., ref. [71]).

2. The ratio

$$
\frac{\mathcal{M}_{(h h)}(s, t)}{\mathcal{M}_{(h h)}(s, 0)} \underset{s \rightarrow \infty}{\sim} \frac{2 J_{1}(\varrho)}{\varrho}
$$


depends on $s$ and $t$ only through the combination $\varrho \sim \mu^{-1} \sqrt{-t} \log s$, with $\mu=$ $\tilde{m} /(\tilde{s}-1)$. Furthermore, the function

$$
f(\tau)=\lim _{s \rightarrow \infty} \frac{\mathcal{M}_{(h h)}\left(s,-\mu^{2} \tau \chi^{-2}\right)}{\mathcal{M}_{(h h)}(s, 0)}=\frac{2 J_{1}(\sqrt{\tau})}{\sqrt{\tau}}
$$

is entire of order $1 / 2$, in agreement with a theorem by Auberson, Kinoshita and Martin [72].

3. At high energy, the elastic scattering amplitude is a universal function of the form

$$
\mathcal{M}_{(h h)}(s, t) \sim i s \log ^{2} \frac{s}{m_{1} m_{2}} M\left(\sqrt{-t} \log \frac{s}{m_{1} m_{2}}\right),
$$

where we have straightforwardly extended the result to the case of colliding particles of different mass.

The well-known results of the black-disk model therefore hold: in particular, one can show that $^{18} \sigma_{\mathrm{el}}^{(h h)}=\sigma_{\text {tot }}^{(h h)} / 2$, and that the $B$-slope at zero transferred momentum,

$$
\mathcal{B}(s) \equiv \frac{d}{d t}\left[\log \frac{d \sigma_{\mathrm{el}}^{(h h)}}{d t}\right]_{t=0},
$$

satisfies the relation $8 \pi \mathcal{B}(s)=\sigma_{\text {tot }}^{(h h)}$. Moreover, the differential elastic cross section vanishes for $t_{0}$ satisfying $\left|t_{0}\right|(\eta / \tilde{m})^{2}=x_{0}^{2}$, where $x_{0} \simeq 3.83$ is the first zero of the function $J_{1}(x)$. Identifying $t_{0}$ with the position $t_{\text {dip }}$ of the dip seen in differential elastic cross sections, one expects that at high energy $\left|t_{\text {dip }}\right| \sigma_{\text {tot }}^{(h h)}=2 \pi x_{0}^{2}$. Notice that experimental data give $\sigma_{\text {el }}^{(h h)} / \sigma_{\text {tot }}^{(h h)} \simeq 0.26$ and $8 \pi \mathcal{B}(s) / \sigma_{\text {tot }}^{(h h)} \simeq 1.97$ at $\sqrt{s}=7 \mathrm{TeV}$, and $\sigma_{\text {el }}^{(h h)} / \sigma_{\text {tot }}^{(h h)} \simeq 0.27$ at $\sqrt{s}=8 \mathrm{TeV}[1-4]$. Furthermore, recent analyses [76, 77] show that $\left|t_{\text {dip }}\right| \sigma_{\text {tot }}^{(h h)} /\left(2 \pi x_{0}^{2}\right)$ is well above 1 up to LHC energies. If the black-disk picture is correct, this indicates that the asymptotic region is still far away from the energies presently available at colliders.

Finally, one can uncover the nature of the Pomeron singularity in the complex angular momentum plane at high energy, by means of the Mellin transform,

$$
\overline{\mathcal{M}}_{(h h)}(\omega, t)=\int_{m^{2}}^{\infty} \frac{d s}{s}\left(\frac{s}{m^{2}}\right)^{-\omega} \mathcal{M}_{(h h)}(s, t)
$$

From eq. (5.36) one easily obtains

$$
\overline{\mathcal{M}}_{(h h)}(\omega, t)=\frac{4 \pi i\left(\frac{m}{\mu}\right)^{2}}{\left[(\omega-1)^{2}-\frac{t}{\mu^{2}}\right]^{\frac{3}{2}}},
$$

which already appeared in ref. [78]. This shows that the Pomeron singularity at positive $t$ is not a pole, but rather an algebraic singularity, while at $t=0$ it is a triple pole. The position of the singularity is

$$
\omega_{\mathbb{P}}^{ \pm}=1 \pm \frac{\sqrt{t}}{\mu}
$$

i.e., the Pomeron trajectory is nonlinear.

\footnotetext{
${ }^{18}$ This requires to use the expression eq. (5.36) in the whole range $t \in[-\infty, 0]$, which can be justified assuming that the small- $t$ region gives the dominant contribution to the elastic cross section.
} 


\section{Conclusions}

In this paper we have shown how to obtain the leading energy dependence of hadronic total cross sections, in the framework of the nonperturbative approach to soft high-energy scattering based on Wilson-loop correlation functions [38-42], if certain nontrivial analyticity assumptions are satisfied. The total cross sections turn out to be of "Froissart" type, $\sigma_{\text {tot }}^{(h h)}(s) \sim B \log ^{2} s$ for $s \rightarrow \infty$. We have also discussed a few scenarios in which the coefficient $B$ turns out to be universal, i.e., independent of the hadrons involved in the scattering process.

Our results rely mainly on the possibility of expressing the Wilson-loop correlator, at high energy and large impact parameter $b$, as a function of the combination

$$
w(\chi, b)=e^{\chi(\tilde{s}-1)} \frac{e^{-b \tilde{m}}}{\sqrt{b \tilde{m}}}
$$

where $\chi \simeq \log \left(s / m^{2}\right)$, and $\tilde{s}$ and $\tilde{m}$ are respectively the spin and mass of the particle that maximises the ratio $l_{0}^{(a)}=\left(s^{(a)}-1\right) / m^{(a)}$ over the asymptotic one-particle states $a$.

The "natural" scenarios leading to universality of $B$ depend on the large- $w$ behaviour of the Wilson-loop correlator (WLC). We have discussed three possibilities: (1) WLC $\rightarrow 0$ as $w \rightarrow \infty$, (2) WLC oscillates as $w \rightarrow \infty$, and (3) WLC $\rightarrow$ const. as $w \rightarrow \infty$. In cases 1 and 2, $B$ turns out to be entirely determined by the hadronic spectrum, and reads $B_{\mathrm{th}}^{(1,2)}=\frac{2 \pi}{\mu^{2}}$, where $\mu=\tilde{m} /(\tilde{s}-1)$. In case 3 , analyticity and crossing symmetry require that the constant is real, and one finds that $B_{\mathrm{th}}^{(3)}=\kappa B_{\mathrm{th}}^{(1,2)}$, with $0 \leq \kappa \leq 2$ due to unitarity; $\kappa$ is however not determined at this stage.

Although the precise form of $w(\chi, b)$ in eq. (6.1) depends on some technical assumptions on certain matrix elements of the relevant Wilson loops, a more general form $\bar{w}(\chi, b)=$ $e^{\chi c} e^{-b M} /(b M)^{(1+\lambda) / 2}$ still holds if these assumptions are relaxed. In this more general case, one finds $B_{\text {th }}^{(1,2) \text { gen }}=\frac{2 \pi c^{2}}{M^{2}}$, which is independent of $\lambda$; one however loses the simple connection with the spectrum. We note in passing that the possibility to express the Wilson-loop correlator as a function of $\bar{w}(\chi, b)$ for some values of $c, M$ and $\lambda$ can be made into a general assumption, independently of our derivation: this would obviously lead to the same results discussed in this paper.

In our calculation, we found that the first subleading correction in energy is proportional to $\log s \cdot \log \log s$. The approach to soft high-energy scattering based on Wilson-loop correlation functions is expected to correctly yield the leading energy dependence of scattering amplitudes and total cross sections, while it is not clear how trustworthy the subleading terms are: indeed, to settle this question one should carefully estimate the energy dependence of the subleading terms discarded in the derivation of eqs. (2.1) and (2.6) (see refs. $[36,38,39,60])$. Nevertheless, a $\log s \cdot \log \log s$ term has never been considered so far in fits to the experimental data, and we believe it worth to include it in a systematic analysis of total cross sections. Such an analysis is however beyond the scope of this paper: we have only checked that in fits limited to the high energy region only $(\chi \gtrsim 5 \div 7)$ the resulting 


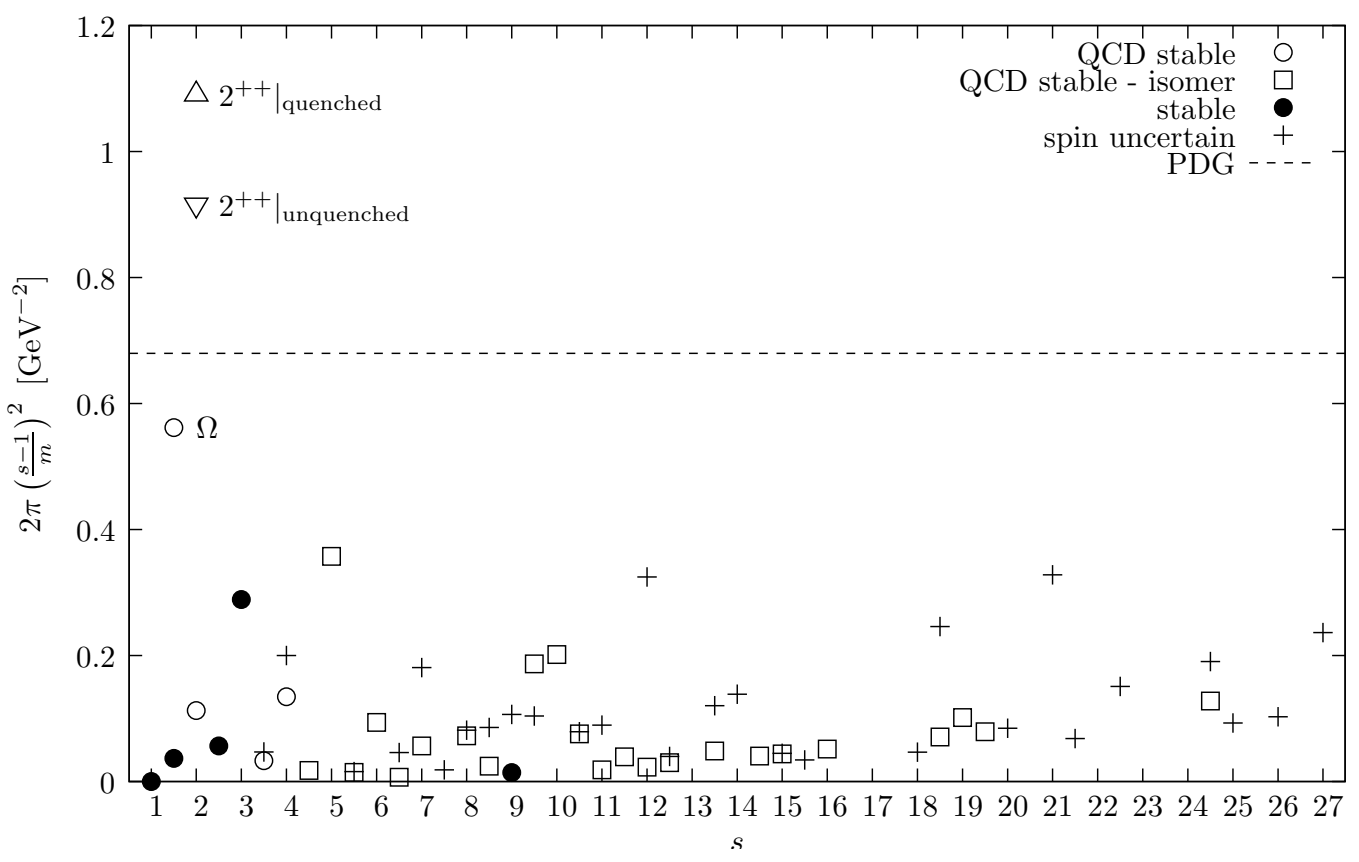

Figure 3. Value of the ratio $2 \pi\left(\frac{s-1}{m}\right)^{2}$ for QCD-stable one-particle states, plotted against the spin $s$, for $s \geq 1$. Only light mesons and baryons, and ground states and long-lived isomers of nuclei have been considered, and only the lightest states are shown for a given spin. Nuclear data are taken from ref. [79]. The maximal value of $2 \pi\left(\frac{s-1}{m}\right)^{2}$ provides the coefficient $B_{\text {th }}$ of the $\log ^{2} s$ term in $\sigma_{\text {tot }}^{(h h)}$ in the scenarios 1 and 2 discussed in the text. The value of $B_{\exp }$ reported by the Particle Data Group [17], and the value of $B_{\text {th }}$ obtained from the $2^{++}$glueball (data taken from refs. [80, 83]), are also shown for comparison.

value of $B$ is not very much affected by its presence. ${ }^{19}$ Indeed, $B$ slightly increases, as expected, when this extra term is included, but the change is smaller than the errors. Our expectation is therefore that the value of $B$ would not change much also in a systematic analysis, so that we can compare the theoretical prediction for $B$ with the value currently reported by the Particle Data Group [17],

$$
B_{\exp }=\frac{2 \pi}{M^{2}}, \quad M=3.04(3) \mathrm{GeV},
$$

i.e., $B_{\exp } \simeq 0.680(13) \mathrm{GeV}^{-2}[0.2646(49) \mathrm{mb}]$.

As we have said above, the theoretical expectation for $B$ in our "favourite" scenarios 1 and 2 is obtained from the spectrum of stable particles with spin larger than 1 , but one has to clarify what "stable" means in this context. As only strong interactions have been considered in the derivation of the basic formula for hadronic scattering amplitudes, eqs. (2.1) and (2.6), a state has to be considered "stable" if it is so when considering QCD in isolation. Among mesons and baryons with baryonic number 1 , only the $\Omega^{ \pm}$is stable and with large enough spin. The $\Omega^{ \pm}$baryon $\left(m_{\Omega^{ \pm}} \simeq 1.67 \mathrm{GeV}\right)$ has quantum numbers

\footnotetext{
${ }^{19}$ The function used for our checks is of the form $\sigma_{\text {tot }}^{(h h)}=B \eta^{2}+C \eta+D$, approximating $\eta$ using its large- $\chi$ expansion, eq. (5.15).
} 
$J^{P}=\frac{3}{2}^{+}$, electric charge $|Q|=1$ and strangeness $|S|=3$. The other known QCD-stable states of high spin are nuclear states. A comprehensive study of nuclei is beyond the scope of this paper: here we limit ourselves to the nuclear ground states and long-lived isomers ${ }^{20}$ that are stable in Nature or decay through the electroweak interactions. As can be seen in figure 3 (data for nuclear states are taken from ref. [79]), the $\Omega^{ \pm}$maximises the relevant ratio, and yields $B_{\text {th }} \simeq 0.56 \mathrm{GeV}^{-2}(0.22 \mathrm{mb})$, which is in fair agreement with experiments, being about $20 \%$ smaller than the value eq. (6.2) reported by the Particle Data Group. In the comparison one should take into account that the values of $B_{\exp }$ reported in refs. [917] show a variation of around $10 \%$ due to different fitting procedures, which suggests a corresponding systematic error.

Strictly speaking, in this paper we have discussed meson-meson scattering, starting from dipole-dipole scattering, while experimental data for total cross sections are available mainly for baryon-baryon and meson-baryon scattering. However, adopting a quarkdiquark picture for baryons (see refs. [38-42, 61]), our results extend immediately to these processes. Moreover, the arguments of section 4 can be easily generalised to more complicated Wilson loops, aimed at describing the three-body structure of baryons, or the inclusion of gluons and sea quarks in the description of hadrons. Under the same assumptions made in this paper, one arrives at the same behaviour of total cross sections obtained here, i.e., "Froissart-like" total cross sections; also, the same considerations can be made concerning universality.

It is also worth noting, at this point, that the description of hadrons in terms of dipoles is (probably) most naturally justified in the quenched limit (or in the limit of a large number of colours $N_{c}$ ), which would lead to consider the quenched (i.e., pure-gauge) theory as the relevant one. In this case, the relevant spectrum would be the glueball spectrum, and, in particular, the spectrum of stable glueballs with spin larger than 1, which, according to ref. [80], are those with $J^{P C}=2^{++}, 2^{+-}, 2^{-+}, 2^{--}, 3^{++}, 3^{+-}, 3^{--}$. Always according to ref. [80] (but see also refs. [81, 82] for other more recent quenched determinations of the glueball spectrum), among the glueballs with $J=2$, the lightest (and thus relevant) one is the $2^{++}$, with $M_{2^{++}}^{(Q)} \simeq 2.40 \mathrm{GeV}$, while the lightest glueball with $J=3$ is the $3^{+-}$, with $M_{3^{+-}}^{(Q)} \simeq 3.55 \mathrm{GeV} .{ }^{21}$ They would lead to a value of the coefficient $B$ given by, respectively, $B_{2^{++}}^{(Q)} \simeq 1.09 \mathrm{GeV}^{-2}(0.42 \mathrm{mb})$ and $B_{3^{+-}}^{(Q)} \simeq 1.99 \mathrm{GeV}^{-2}(0.77 \mathrm{mb})$. Therefore, according to our approach, we should conclude that, in the quenched theory, $B_{\mathrm{th}}^{(Q)}=B_{3^{+-}}^{(Q)} \simeq 1.99 \mathrm{GeV}^{-2}$ (0.77 mb), which is (quite surprisingly) a factor 3 larger than $B_{\exp }$.

An interesting issue is the possible effectiveness of one-particle selection rules to reduce the set of states over which $l_{0}^{(a)}$ has to be maximised. It is rather easy to prove selection rules for spin $\left(s_{3}\right)$, parity $\left(\eta_{P}\right)$ and charge conjugation $\left(\eta_{C}\right)$ for the one-particle contributions: they are nonzero only if $\eta_{P}=\eta_{C}=e^{i \pi s_{3}}$. But even if a particle does not contribute

\footnotetext{
${ }^{20}$ A detailed study should include all the excited nuclear states that are QCD-stable.

${ }^{21}$ We would like to point out, at this point, that the identification of the spin of glueball states on the lattice is highly nontrivial and, in some cases (such as $2^{+-}, 2^{--}, 3^{++}$and $3^{--}$), also controversial [80]. Moreover, a more recent (unquenched) determination of the glueball spectrum [83] has not found evidence for the states $2^{+-}, 3^{++}$and $3^{--}$, leaving, however, the states $2^{++}$and $3^{+-}$as possible relevant states, with masses $M_{2^{++}} \simeq 2.62 \mathrm{GeV}$ and $M_{3+-} \simeq 3.85 \mathrm{GeV}$.
} 
at this level, there is no reason for it not to contribute starting from the two-particle level, so that it should be included in the set over which $l_{0}^{(a)}$ has to be maximised. Things could change if the (connected) contributions of many-particle states were suppressed by additional powers of $e^{\chi}$, but we have not found any argument supporting this possibility. If, for some reason, states for which one-particle contributions are nonzero should be considered to be "dominant", then (see also footnote 21 ) we would be left with the glueball $2^{++}$, with $B_{\mathrm{th}}^{(Q)}=B_{2^{++}}^{(Q)} \simeq 1.09 \mathrm{GeV}^{-2}(0.42 \mathrm{mb})$, as it has been already suggested in ref. [84], commenting the results of the best fits to the lattice data performed in ref. [59]. ${ }^{22}$ This value is still larger than $B_{\text {exp }}$, but "only" by a factor 1.6 [and an even better agreement with $B_{\text {exp }}$ would be obtained if we used the unquenched value $M_{2^{++}} \simeq 2.62 \mathrm{GeV}$ found in ref. [83], which leads to $\left.B_{2^{++}} \simeq 0.91 \mathrm{GeV}^{-2}(0.35 \mathrm{mb})\right]$. Therefore, a cautious conclusion could be that, in the quenched theory, $B_{\mathrm{th}}^{(Q)}$ is at least $1.09 \mathrm{GeV}^{-2}(0.42 \mathrm{mb})$. The comparison with the unquenched estimate $B_{\mathrm{th}} \simeq 0.56 \mathrm{GeV}^{-2}(0.22 \mathrm{mb})$, that we have found above, ${ }^{23}$ seems to suggest (quite surprisingly) large unquenching effects due to the sea quarks. Of course, also the possibility that the relevant state (which maximises the ratio $l_{0}^{(a)}=\frac{s^{(a)}-1}{m^{(a)}}$ ) has not yet been discovered, cannot be excluded.

\section{Acknowledgments}

M.G. is supported by the Hungarian Academy of Sciences under "Lendület" grant No. LP2011-011.

\section{A Technical details}

\section{A.1 Vanishing Wilson-loop matrix elements and massless particles}

In this appendix we briefly discuss the consequences of vanishing matrix elements $\mathcal{F}_{\alpha}\left(\{0\}_{\alpha} ; \nu_{1}\right)$ and/or $\overline{\mathcal{F}}_{\alpha}\left(\{0\}_{\alpha} ; \nu_{2}\right)$ on the large- $\chi$ behaviour of the relevant Wilson-loop correlator, and on the asymptotic behaviour of total cross sections. Furthermore, we discuss the possible effects due to the presence of massless particles in the spectrum.

Let us assume that

$$
\mathcal{F}_{\alpha}\left(\left\{p_{3}\right\}_{\alpha} ; \nu_{1}\right) \overline{\mathcal{F}}_{\alpha}\left(\left\{p_{3}\right\}_{\alpha} ; \nu_{2}\right) \simeq k_{\alpha} \prod_{a, n_{a}(\alpha) \neq 0} \prod_{i=1}^{n_{a}(\alpha)}\left(p_{3}^{(a) i}\right)^{\lambda_{\alpha}^{(a)}}
$$

with $k_{\alpha}$ some function of $\nu_{i}$, and where some of the $\lambda_{\alpha}^{(a)}$ can be zero. One immediately finds after the change of variables eq. (4.36) that in the limit of large $b$ the contribution

\footnotetext{
${ }^{22}$ We note in passing that a Pomeron of gluonic nature and effectively of spin 2 has been recently proposed in ref. [85].

${ }^{23}$ Since the $\Omega^{ \pm}$baryon does not satisfy the selection rules on baryon number, electric charge and strangeness, the above-mentioned (hypothetical) suppression mechanism would make this value into an upper bound.
} 
$\delta C_{\alpha}$ of state $\alpha$ to $C_{n}$ is proportional to

$$
\delta C_{\alpha} \propto \delta_{\mathcal{N}_{\alpha}, n} \prod_{a}\left(\frac{1}{\left(b m^{(a)}\right)^{\frac{1+\lambda_{\alpha}^{(a)}}{2}}} e^{\chi\left[s^{(a)}-1\right]} e^{-b m^{(a)}}\right)^{n_{a}(\alpha)} .
$$

Here $\lambda_{\alpha}^{(a)}$ is the same for all particles of the same species due to symmetry reasons. As anticipated in section 4.4 , the only effect of vanishing $\mathcal{F}_{\alpha}, \overline{\mathcal{F}}_{\alpha}$ on the large- $b$ behaviour of $\delta C_{\alpha}$ is the appearence of extra inverse powers of $b$.

The quantities $\mathcal{F}_{\alpha}\left(\{0\}_{\alpha} ; \nu_{1}\right)$ and $\overline{\mathcal{F}}_{\alpha}\left(\{0\}_{\alpha} ; \nu_{2}\right)$ are actually expected to vanish in the presence of massless particles, since in this case the phase-space measure contains factors $d p_{3} / p_{3}$, and the integral would diverge otherwise. Including massless particles requires only a minor modification to the calculation of section 4.4 , and we obtain for $\delta C_{\alpha}$ in the limit of large $b$

$$
\delta C_{\alpha} \propto \delta_{\mathcal{N}_{\alpha}, n} \prod_{a}\left(\frac{1}{\left(b m^{(a)}\right)^{\frac{1+\lambda_{\alpha}^{(a)}}{2}}} e^{\chi\left[s^{(a)}-1\right]} e^{-b m^{(a)}}\right)^{n_{a}(\alpha)} \prod_{a^{\prime}}\left(\frac{1}{b^{\lambda_{\alpha}^{\left(a^{\prime}\right)}}} e^{\chi\left[s^{\left(a^{\prime}\right)}-1\right]}\right)^{n_{a^{\prime}}(\alpha)}
$$

where the indices $a$ and $a^{\prime}$ run over massive and massless particles, respectively.

The modifications discussed above affect the asymptotic expression eq. (4.49) for the relevant Wilson-loop correlator. If only massive particles are present, one has simply to introduce the extra powers of $b$ discussed above, finding

$$
C_{n}\left(-i \chi ; b ; \nu_{1}, \nu_{2}\right) \underset{\chi \rightarrow \infty}{\sim} \frac{(i z)^{n}}{\left[2 \pi \log \left(\frac{e^{(\tilde{s}-1) \chi}}{z}\right)^{1+\lambda}\right]^{\frac{n}{2}}} \bar{C}_{n}^{0}\left(\nu_{1}, \nu_{2}\right) \equiv\left(\frac{i w_{\lambda}}{\sqrt{2 \pi}}\right)^{n} \bar{C}_{n}^{0}\left(\nu_{1}, \nu_{2}\right),
$$

with $\lambda$ being the appropriate power corresponding to the relevant particle(s). ${ }^{24}$ On the other hand, in the presence of massless particles, one has to reconsider the procedure leading to eq. (4.49). If a state contains massless particles, its contribution to $C_{n}$ takes the form eq. (A.3). It is easy to see that if $(\tilde{s}-1) / \tilde{m}$ can be maximised over the massive particles (yielding $\tilde{s}>1$ ), changing variables to $z$, eq. (5.9), one gets extra factors from each massless particle of the form

$$
\left(\frac{e^{\chi\left(s^{(0)}-1\right)}}{b^{\lambda^{(0)}}}\right)^{n^{(0)}}=\left(\frac{e^{\chi\left(s^{(0)}-1\right)}}{\left[\frac{1}{\tilde{m}}(\chi(\tilde{s}-1)-\log z)\right]^{\lambda^{(0)}}}\right)^{n^{(0)}} .
$$

If $s^{(0)}=0$, eq. (A.5) vanishes exponentially in $\chi$ at high energy, meaning that scalar massless particles can be safely neglected; this allows to safely consider the chiral limit of QCD. If $s^{(0)}=1$, on the other hand, eq. (A.5) vanishes only as a power of $\chi$, so that it can

\footnotetext{
${ }^{24}$ For simplicity, we are assuming that $\lambda$ is $n$-independent. If $\lambda$ depends on $n$, only those terms with smallest $\lambda$ have to be kept to leading order in $\chi$. Also, eq. (A.4) holds only for $n=2 k$ if we are in case 2 discussed in section 4.5 , while $C_{2 k+1}$ is suppressed exponentially in $\chi$.
} 
give important subleading contributions. ${ }^{25}$ The situation is drastically different if massless particles with $s^{(0)} \geq 2$ are present: in this case the proper change of variable is rather $z=e^{\chi\left(s^{(0)}-1\right)} / b^{\lambda^{(0)}}$, with $s^{(0)}$ and $\lambda^{(0)}$ corresponding to the massless particle maximising the ratio $(s-1) / \lambda$, which kills all the massive contributions, and all the other massless contributions as well. We will not consider this case any longer.

The modifications discussed above have only mild consequences on the asymptotic behaviour of the total cross section. To see this, one has simply to repeat the calculation of section 5.1, taking into account that now $\mathcal{C}_{M}\left(\chi ; \vec{b}_{\perp} ; \nu_{1}, \nu_{2}\right) \sim \bar{g}\left(w_{\lambda} ; \nu_{1}, \nu_{2}\right)-1$ for $\chi \rightarrow \infty$, where

$$
w_{\lambda}(\chi, z)=z\left[\log \left(\frac{e^{(\tilde{s}-1) \chi}}{z}\right)\right]^{-\frac{1+\lambda}{2}},
$$

$z=e^{(\tilde{s}-1) \chi} e^{-\tilde{m} b}$ is the same as in eq. (5.9), and $\bar{g}$ is a bounded function, which can be proved exploiting unitarity as in eq. (5.6). Rescaling $z=\xi(\chi) z^{\prime}$, with $\xi(\chi)$ defined by

$$
w_{\lambda}(\chi, z)=w_{\lambda}\left(\chi, \xi(\chi) z^{\prime}\right) \equiv \frac{z^{\prime}}{\left[1+\frac{\log \left(\frac{1}{z^{\prime}}\right)}{\log \left(\frac{e^{(\xi-1) \chi}}{\xi(\chi)}\right)}\right]^{\frac{1+\lambda}{2}}},
$$

setting again $\eta=\log \left(\frac{e^{(\tilde{s}-1) \chi}}{\xi(\chi)}\right)$, and solving the equation for $\xi$, one finds

$$
\eta=\tilde{\lambda} W\left(\frac{1}{\tilde{\lambda}} e^{\frac{1}{\tilde{\lambda}} \chi(\tilde{s}-1)}\right)
$$

with $\tilde{\lambda}=(1+\lambda) / 2$. One then proceeds as in section 5.1 , and if $\Delta=0$ [see eq. (5.23)] one again obtains eq. (5.33), with $\eta$ defined now in eq. (A.8). The leading term in the expansion of $\eta$ at large $\chi$ is unchanged, while the first subleading correction is modified,

$$
\eta=(\tilde{s}-1) \chi-\frac{1}{2} \log [(\tilde{s}-1) \chi] \rightarrow(\tilde{s}-1) \chi-\tilde{\lambda} \log [(\tilde{s}-1) \chi] .
$$

As a consequence, also for nonzero $\lambda$ the total cross section is of the form eq. (5.34), with an extra factor of $\tilde{\lambda}$ in front of the coefficient of the subleading $\log s \cdot \log \log s$ term, but with exactly the same leading term.

Finally, let us make contact with the parameterisations of lattice data discussed in ref. [59]. The functional forms considered there for the Euclidean correlator, after analytic continuation to Minkowski space and in the large- $\chi$ limit, reduce to the general form

$$
\begin{aligned}
& \mathcal{C}_{M}\left(\chi ; \vec{b}_{\perp} ; \nu_{1}, \nu_{2}\right) \underset{\chi \rightarrow \infty}{\simeq} \exp \left\{\mathcal{K}_{M}\left(\chi ; \vec{b}_{\perp} ; \nu_{1}, \nu_{2}\right)\right\}-1, \\
& \mathcal{K}_{M}\left(\chi ; \vec{b}_{\perp} ; \nu_{1}, \nu_{2}\right)=i \beta\left(\nu_{1}, \nu_{2}\right) \chi^{p} e^{n \chi} e^{-\mu b} .
\end{aligned}
$$

They are therefore functions of the variable $\tilde{w}=\chi^{p} \tilde{z}$ only, with $\tilde{z}=e^{n \chi} e^{-\mu b}$, which, up to subleading terms at large $\chi$, coincides with eq. (A.6) upon identifying $n=\tilde{s}-1, \mu=\tilde{m}$ and $p=-(1+\lambda) / 2$. These parameterisations therefore lead to the same high-energy behaviour

\footnotetext{
${ }^{25}$ In principle these contributions could also be dominant, if $\lambda^{(0)}$ were smaller than the power corresponding to the relevant massive particles.
} 
of total cross sections found here, as already discussed in ref. [59]. Furthermore, if $\operatorname{Im} \beta>0$, they satisfy our first criterion for universality, i.e., vanishing of the Wilson-loop correlator at large $\chi$ and fixed $b$, while if $\operatorname{Im} \beta=0$ the correlator oscillates wildly at large $\chi$ and fixed $b$, thus satisfying our second criterion for universality.

\section{A.2 Resummation for factorised matrix elements: general case}

We want now to extend the discussion of section 4.5 concerning the possibility to interchange the order of summation and analytic continuation to the case in which particles of type 2 (see section 4.5) are present, when the Wilson-loop matrix elements are approximately in factorised form. Including this kind of particles, the factorised form of the matrix elements reads ${ }^{26}$

$$
\begin{aligned}
& W_{\alpha}\left(\{\vec{p}\}_{\alpha},\left\{s_{3}\right\}_{\alpha} ; \nu_{1}\right) \simeq \\
& \prod_{a, n_{a}(\alpha) \neq 0}^{(1)} \prod_{i=1}^{n_{a}(\alpha)} \lim _{T \rightarrow \infty} \frac{\left\langle 0\left|\hat{\mathcal{W}}_{E}\left[\tilde{\mathcal{C}}_{0}^{(T)}\left(\nu_{1}\right)\right]\right| \alpha, \vec{p}^{(a) i}, s_{3}^{(a) i} ; \text { in }\right\rangle}{\left\langle 0\left|\hat{\mathcal{W}}_{E}\left[\tilde{\mathcal{C}}_{0}^{(T)}\left(\nu_{1}\right)\right]\right| 0\right\rangle} \\
& \times \prod_{a, n_{a}(\alpha) \neq 0}^{(2)} \sum_{P_{a}} \prod_{i=1}^{n_{a}(\alpha)} \lim _{T \rightarrow \infty} \frac{\left\langle 0\left|\hat{\mathcal{W}}_{E}\left[\tilde{\mathcal{C}}_{0}^{(T)}\left(\nu_{1}\right)\right]\right| \alpha, \vec{p}^{(a) i}, \vec{p}^{(\bar{a}) i_{P a}}, s_{3}^{(a) i}, s_{3}^{(\bar{a}) i_{P_{a}}} ; \text { in }\right\rangle}{\left\langle 0\left|\hat{\mathcal{W}}_{E}\left[\tilde{\mathcal{C}}_{0}^{(T)}\left(\nu_{1}\right)\right]\right| 0\right\rangle} \\
& \equiv \prod_{a, n_{a}(\alpha) \neq 0}^{(1)} \prod_{i=1}^{n_{a}(\alpha)} W_{a}\left(\vec{p}^{(a) i}, s_{3}^{(a) i} ; \nu_{1}\right) \\
& \times \prod_{a, n_{a}(\alpha) \neq 0}^{(2)} \sum_{P_{a}} \prod_{i=1}^{n_{a}(\alpha)} W_{a \bar{a}}\left(\vec{p}^{(a) i}, \vec{p}^{(\bar{a}) i_{P_{a}}}, s_{3}^{(a) i}, s_{3}^{(\bar{a}) i_{P_{a}}} ; \nu_{1}\right),
\end{aligned}
$$

where $W_{a}$ are one-particle matrix elements, and $W_{a \bar{a}}$ are particle-antiparticle pair matrix elements; a similar result holds for $\bar{W}_{\alpha}$. Here the superscript (1) and (2) indicate that the products in eq. (A.11) are restricted to particles of type 1 and particle-antiparticle pairs of type 2 , respectively; $\bar{a}$ denotes the antiparticle of particle $a ; P_{a}$ is a permutation of $1, \ldots, n_{a}(\alpha)$ clearly $n_{a}(\alpha)=n_{\bar{a}}(\alpha)$. From eqs. (4.13) and (4.16), and using the multinomial theorem, we get

$$
\begin{aligned}
& \tilde{C}_{E} \simeq \exp \{\left\{\sum_{a}^{(1)} \sum_{s_{3}=-s^{(a)}}^{s^{(a)}} e^{i \theta s_{3}} \int d \Omega_{a} e^{-b \varepsilon^{(a)}} W_{a}\left(\mathcal{R}_{\frac{\theta}{2}} \vec{p}_{a}, s_{3} ; \nu_{1}\right) \bar{W}_{a}\left(\mathcal{R}_{-\frac{\theta}{2}} \vec{p}_{a}, s_{3} ; \nu_{2}\right)\right. \\
&+ \frac{1}{2} \sum_{a}^{(2)} \sum_{s_{3}=-s^{(a)}}^{s^{(a)}} \sum_{\bar{s}_{3}=-s^{(a)}}^{s^{(a)}} e^{i \theta\left(s_{3}+\bar{s}_{3}\right)} \int d \Omega_{a} d \Omega_{\bar{a}} e^{-b\left[\varepsilon^{(a)}+\varepsilon^{(\bar{a})}\right]} \\
&\left.\quad \times W_{a \bar{a}}\left(\mathcal{R}_{\frac{\theta}{2}} \vec{p}_{a}, \mathcal{R}_{\frac{\theta}{2}} \vec{p}_{\bar{a}}, s_{3}, \bar{s}_{3} ; \nu_{1}\right) \bar{W}_{a \bar{a}}\left(\mathcal{R}_{-\frac{\theta}{2}} \vec{p}_{a}, \mathcal{R}_{-\frac{\theta}{2}} \vec{p}_{\bar{a}}, s_{3}, \bar{s}_{3} ; \nu_{2}\right)\right\}-1,
\end{aligned}
$$

\footnotetext{
${ }^{26}$ Here we are assuming that a particle can be paired only to its antiparticle to evade the selection rules. Dropping this assumption would only make the combinatorics more complicated, without affecting the argument.
} 
where $d \Omega_{a}=d^{3} p_{a} /\left[(2 \pi)^{3} 2 \varepsilon^{(a)}\right]$ is the phase-space element for a particle of type $a$, and $\varepsilon^{(a)}$ the corresponding energy. The argument then goes as in section 4.5: the sums in the exponent in eq. (A.12) are over finite sets, so that there is no convergence problem, and one can verify explicitly that analytic continuation and summation over the complete set of states commute.

Open Access. This article is distributed under the terms of the Creative Commons Attribution License (CC-BY 4.0), which permits any use, distribution and reproduction in any medium, provided the original author(s) and source are credited.

\section{References}

[1] TOTEM collaboration, G. Antchev et al., First measurement of the total proton-proton cross section at the LHC energy of $\sqrt{s}=7$ TeV, Europhys. Lett. 96 (2011) 21002 [arXiv:1110.1395] [INSPIRE].

[2] TOTEM collaboration, G. Antchev et al., Measurement of proton-proton elastic scattering and total cross-section at $\sqrt{s}=7$ TeV, Europhys. Lett. 101 (2013) 21002.

[3] TOTEM collaboration, G. Antchev et al., Luminosity-independent measurements of total, elastic and inelastic cross-sections at $\sqrt{s}=7$ TeV, Europhys. Lett. 101 (2013) 21004.

[4] TOTEM collaboration, G. Antchev et al., Luminosity-Independent Measurement of the Proton-Proton Total Cross Section at $\sqrt{s}=8$ TeV, Phys. Rev. Lett. 111 (2013) 012001 [INSPIRE].

[5] A. Kohara, E. Ferreira and T. Kodama, Amplitudes and Observables in pp Elastic Scattering at $\sqrt{s}=7$ TeV, Eur. Phys. J. C 73 (2013) 2326 [arXiv:1212.3652] [INSPIRE].

[6] I. Dremin, Interplay of total cross sections and ratios of real to imaginary parts of hadron amplitudes, JETP Lett. 97 (2013) 571 [arXiv:1304.5345] [INSPIRE].

[7] I. Dremin and V. Nechitailo, Proton periphery activated by multiparticle dynamics, Nucl. Phys. A 916 (2013) 241 [arXiv:1306.5384] [InSPIRE].

[8] E. Gotsman, E. Levin and U. Maor, Description of LHC data in a soft interaction model, Phys. Lett. B 716 (2012) 425 [arXiv:1208.0898] [InSPIRE].

[9] K. Igi and M. Ishida, Investigations of the $\pi N$ total cross-sections at high-energies using new FESR: $\log (\nu)$ or $\log ^{2}(\nu)$, Phys. Rev. D 66 (2002) 034023 [hep-ph/0202163] [INSPIRE].

[10] J. Cudell, V. Ezhela, P. Gauron, K. Kang, Y. Kuyanov et al., Hadronic scattering amplitudes: Medium-energy constraints on asymptotic behavior, Phys. Rev. D 65 (2002) 074024 [hep-ph/0107219] [INSPIRE].

[11] K. Igi and M. Ishida, Predictions of $p p, \bar{p} p$ total cross section and $\rho$ ratio at LHC and cosmic-ray energies, Phys. Lett. B 622 (2005) 286 [hep-ph/0505058] [INSPIRE].

[12] M. Block and F. Halzen, New evidence for the saturation of the Froissart bound, Phys. Rev. D 72 (2005) 036006 [Erratum ibid. D 72 (2005) 039902] [hep-ph/0506031] [INSPIRE].

[13] Particle Data Group collaboration, W. Yao et al., Review of Particle Physics, J. Phys. G 33 (2006) 1 [INSPIRE].

[14] M. Ishida and K. Igi, Universal Rise of Hadronic Total Cross Sections based on Forward $\pi p$ and $\bar{p} p(p p)$ Scatterings, Phys. Lett. B 670 (2009) 395 [arXiv:0809.2424] [InSPIRE]. 
[15] M. Ishida and K. Igi, Test of universal rise of hadronic total cross sections based on $\pi p, K p$, $\bar{p} p$ and pp scatterings, Prog. Theor. Phys. Suppl. 187 (2011) 297 [InSPIRE].

[16] M.M. Block and F. Halzen, Experimental Confirmation that the Proton is Asymptotically a Black Disk, Phys. Rev. Lett. 107 (2011) 212002 [arXiv:1109.2041] [INSPIRE].

[17] Particle Data Group collaboration, J. Beringer et al., Review of Particle Physics (RPP), Phys. Rev. D 86 (2012) 010001 [inSPIRE].

[18] M. Froissart, Asymptotic behavior and subtractions in the Mandelstam representation, Phys. Rev. 123 (1961) 1053 [INSPIRE].

[19] A. Martin, Extension of the axiomatic analyticity domain of scattering amplitudes by unitarity. 1., Nuovo Cim. A 42 (1965) 930 [inSPIRE].

[20] L. Lukaszuk and A. Martin, Absolute upper bounds for pi pi scattering, Nuovo Cim. A 52 (1967) 122 [INSPIRE].

[21] A. Martin, The Froissart bound for inelastic cross-sections, Phys. Rev. D 80 (2009) 065013 [arXiv: 0904.3724] [INSPIRE].

[22] T.T. Wu, A. Martin, S.M. Roy and V. Singh, An upper bound on the total inelastic cross-section as a function of the total cross-section, Phys. Rev. D 84 (2011) 025012 [arXiv: 1011.1349] [INSPIRE].

[23] A. Martin and S. Roy, Froissart Bound on Total Cross-section without Unknown Constants, arXiv: 1306.5210 [INSPIRE].

[24] D. Greynat and E. de Rafael, The Froissart-Martin Bound for $\pi \pi$ Scattering in QCD, Phys. Rev. D 88 (2013) 034015 [arXiv: 1305.7045] [INSPIRE].

[25] L.L. Jenkovszky, B. Struminsky and A. Vall, Where Is Asymptotics of Hadron Interactions? (in Russian), Yad. Fiz. 46 (1987) 1519 [INSPIRE].

[26] J. Finkelstein, H. Fried, K. Kang and C.-I. Tan, Forward Scattering at Collider Energies and Eikonal Unitarization of Odderon, Phys. Lett. B 232 (1989) 257 [INSPIRE].

[27] G. Başar, D.E. Kharzeev, H.-U. Yee and I. Zahed, Holographic Pomeron and the Schwinger Mechanism, Phys. Rev. D 85 (2012) 105005 [arXiv: 1202.0831] [INSPIRE].

[28] E. Ferreiro, E. Iancu, K. Itakura and L. McLerran, Froissart bound from gluon saturation, Nucl. Phys. A 710 (2002) 373 [hep-ph/0206241] [inSPIRE].

[29] L. Frankfurt, M. Strikman and M. Zhalov, On the universality of cross sections of hadron-hadron (nuclei) collisions at superhigh energies, Phys. Lett. B 616 (2005) 59 [hep-ph/0412052] [INSPIRE].

[30] W. Heisenberg, Production of Mesons as a Shock Wave Problem (in German), Zeitschrift für Physik 133 (1952) 65.

[31] H.G. Dosch, P. Gauron and B. Nicolescu, Heisenberg's universal $l n^{2} s$ increase of total cross-sections, Phys. Rev. D 67 (2003) 077501 [hep-ph/0206214] [INSPIRE].

[32] D. Fagundes, M. Menon and P. Silva, Total Hadronic Cross Section Data and the Froissart-Martin Bound, Braz. J. Phys. 42 (2012) 452 [arXiv:1112.4704] [INSPIRE].

[33] D. Fagundes and M. Menon, Total Hadronic Cross Section and the Elastic Slope: An Almost Model-Independent Connection, Nucl. Phys. A 880 (2012) 1 [arXiv:1112.5115] [INSPIRE]. 
[34] D. Fagundes, M. Menon and P. Silva, On the rise of the proton-proton cross-sections at high energies, J. Phys. G 40 (2013) 065005 [arXiv:1208.3456] [InSPIRE].

[35] Y. Azimov, How Robust is the Froissart Bound?, Phys. Rev. D 84 (2011) 056012 [arXiv:1104.5314] [INSPIRE].

[36] O. Nachtmann, Considerations concerning diffraction scattering in quantum chromodynamics, Annals Phys. 209 (1991) 436 [InSPIRE].

[37] S. Donnachie, G. Dosch, P. Landshoff and O. Nachtmann, Pomeron Physics and QCD, Cambridge University Press, Cambridge, 2002.

[38] H.G. Dosch, E. Ferreira and A. Krämer, Nonperturbative QCD treatment of high-energy hadron hadron scattering, Phys. Rev. D 50 (1994) 1992 [hep-ph/9405237] [INSPIRE].

[39] O. Nachtmann, High-energy collisions and nonperturbative QCD, in Lectures on QCD: Applications, F. Lenz, H.W. Grießhammer and D. Stoll eds., Lect. Notes Phys. 496 (1997) 1 [hep-ph/9609365] [INSPIRE].

[40] E.R. Berger and O. Nachtmann, Differential cross-sections for high-energy elastic hadron hadron scattering in nonperturbative QCD, Eur. Phys. J. C 7 (1999) 459 [hep-ph/9808320] [INSPIRE].

[41] H.G. Dosch, Space-time picture of high energy scattering, in At the frontier of Particle Physics - Handbook of QCD (Boris Ioffe Festschrift), M. Shifman ed., World Scientific, Singapore, 2001, vol. 2, pg. 1195.

[42] A. Shoshi, F. Steffen and H. Pirner, $S$ matrix unitarity, impact parameter profiles, gluon saturation and high-energy scattering, Nucl. Phys. A 709 (2002) 131 [hep-ph/0202012] [INSPIRE].

[43] E. Meggiolaro, The High-energy quark quark scattering: From Minkowskian to Euclidean theory, Z. Phys. C 76 (1997) 523 [hep-th/9602104] [INSPIRE].

[44] E. Meggiolaro, The Analytic continuation of the high-energy quark quark scattering amplitude, Eur. Phys. J. C 4 (1998) 101 [hep-th/9702186] [INSPIRE].

[45] E. Meggiolaro, The Analytic continuation of the high-energy parton-parton scattering amplitude with an IR cutoff, Nucl. Phys. B 625 (2002) 312 [hep-ph/0110069] [INSPIRE].

[46] E. Meggiolaro, On the loop-loop scattering amplitudes in Abelian and non-Abelian gauge theories, Nucl. Phys. B 707 (2005) 199 [hep-ph/0407084] [INSPIRE].

[47] M. Giordano and E. Meggiolaro, A Nonperturbative foundation of the Euclidean-Minkowskian duality of Wilson-loop correlation functions, Phys. Lett. B 675 (2009) 123 [arXiv:0902.4145] [INSPIRE].

[48] A. Shoshi, F. Steffen, H.G. Dosch and H. Pirner, Confining QCD strings, Casimir scaling and a Euclidean approach to high-energy scattering, Phys. Rev. D 68 (2003) 074004 [hep-ph/0211287] [INSPIRE].

[49] E.V. Shuryak and I. Zahed, Instanton induced effects in QCD high-energy scattering, Phys. Rev. D 62 (2000) 085014 [hep-ph/0005152] [INSPIRE].

[50] M. Giordano and E. Meggiolaro, Instanton effects on Wilson-loop correlators: a new comparison with numerical results from the lattice, Phys. Rev. D 81 (2010) 074022 [arXiv:0910.4505] [INSPIRE]. 
[51] R. Janik and R.B. Peschanski, High-energy scattering and the AdS/CFT correspondence, Nucl. Phys. B 565 (2000) 193 [hep-th/9907177] [INSPIRE].

[52] R. Janik and R.B. Peschanski, Minimal surfaces and Reggeization in the AdS/CFT correspondence, Nucl. Phys. B 586 (2000) 163 [hep-th/0003059] [INSPIRE].

[53] R.A. Janik, String fluctuations, AdS/CFT and the soft Pomeron intercept, Phys. Lett. B 500 (2001) 118 [hep-th/0010069] [INSPIRE].

[54] M. Giordano and R. Peschanski, High Energy Bounds on Soft $N=4$ SYM Amplitudes from AdS/CFT, JHEP 05 (2010) 037 [arXiv: 1003.2309] [INSPIRE].

[55] M. Giordano and E. Meggiolaro, High-energy hadron-hadron (dipole-dipole) scattering from lattice QCD, Phys. Rev. D 78 (2008) 074510 [arXiv:0808.1022] [InSPIRE].

[56] E. Meggiolaro and M. Giordano, High-energy hadron-hadron (dipole-dipole) scattering on the lattice, Prog. Theor. Phys. Suppl. 187 (2011) 200 [arXiv:1010.0914] [INSPIRE].

[57] M. Giordano and E. Meggiolaro, Hadron-Hadron Total Cross Sections and Soft High-Energy Scattering on the Lattice, PoS(LATTICE2011) 115 [arXiv:1110.5188] [INSPIRE].

[58] A. Babansky and I. Balitsky, Scattering of color dipoles: From low to high-energies, Phys. Rev. D 67 (2003) 054026 [hep-ph/0212075] [INSPIRE].

[59] M. Giordano, E. Meggiolaro and N. Moretti, Asymptotic Energy Dependence of Hadronic Total Cross Sections from Lattice QCD, JHEP 09 (2012) 031 [arXiv:1203.0961] [INSPIRE].

[60] M. Giordano, Wilson-loop formalism for Reggeon exchange in soft high-energy scattering, JHEP 07 (2012) 109 [Erratum ibid. 1301 (2013) 021] [arXiv:1204.3772] [INSPIRE].

[61] M. Rueter and H.G. Dosch, Nucleon structure and high-energy scattering, Phys. Lett. B 380 (1996) 177 [hep-ph/9603214] [INSPIRE].

[62] M. Giordano and E. Meggiolaro, Analyticity and crossing symmetry of the eikonal amplitudes in gauge theories, Phys. Rev. D 74 (2006) 016003 [hep-ph/0602143] [INSPIRE].

[63] E. Meggiolaro, Can the Pomeron be derived from a Euclidean-Minkowskian duality?, Phys. Lett. B 651 (2007) 177 [hep-ph/0612307] [INSPIRE].

[64] H. Cheng and E.-C. Tsai, Gauge Invariance of the Quantum Wilson Loop, Phys. Rev. D 36 (1987) 3196 [INSPIRE].

[65] S. Weinberg, The Quantum Theory of Fields. Vol. 1: Foundations, Cambridge University Press, Cambridge, 1995.

[66] H. Lehmann, K. Symanzik and W. Zimmermann, On the formulation of quantized field theories, Nuovo Cim. 1 (1955) 205 [INSPIRE].

[67] H. Lehmann, K. Symanzik and W. Zimmermann, On the formulation of quantized field theories. II, Nuovo Cim. 6 (1957) 319 [INSPIRE].

[68] U. Amaldi, M. Jacob and G. Matthiae, Diffraction of Hadronic Waves, Ann. Rev. Nucl. Part. Sci. 26 (1976) 385 [inSPIRE].

[69] R. Castaldi and G. Sanguinetti, Elastic Scattering and Total Cross-section at Very High High-energies, Ann. Rev. Nucl. Part. Sci. 35 (1983) 351 [INSPIRE].

[70] M. Block and R. Cahn, High-Energy pp and pp Forward Elastic Scattering and Total Cross-Sections, Rev. Mod. Phys. 57 (1985) 563 [inSPIRE]. 
[71] R. Eden, Theorems on high energy collisions of elementary particles, Rev. Mod. Phys. 43 (1971) 15 [INSPIRE].

[72] G. Auberson, T. Kinoshita and A. Martin, Violation of the Pomeranchuk theorem and zeros of the scattering amplitudes, Phys. Rev. D 3 (1971) 3185 [inSPIRE].

[73] R. Corless, G. Gonnet, D. Hare, D. Jeffrey and D. Knuth, On the LambertW function, Adv. Comput. Math. 5 (1996) 329 [INSPIRE].

[74] S. Troshin and N. Tyurin, Reflective scattering from unitarity saturation, Int. J. Mod. Phys. A 22 (2007) 4437 [hep-ph/0701241] [INSPIRE].

[75] S. Troshin and N. Tyurin, Beyond the black disk limit, Phys. Lett. B 316 (1993) 175 [hep-ph/9307250] [INSPIRE].

[76] I. Bautista and J. Dias de Deus, The black disk and the dip in the differential elastic cross section at asymptotic energy, Phys. Lett. B 718 (2013) 1571 [arXiv:1212.1764] [INSPIRE].

[77] T. Csörgö and F. Nemes, Elastic scattering of protons from $\sqrt{s}=23.5$ GeV to 7 TeV from a generalized Bialas-Bzdak model, Int. J. Mod. Phys. A 29 (2014) 1450019 [arXiv:1306.4217] [INSPIRE].

[78] P. Gauron, B. Nicolescu and E. Leader, Similarities and Differences Between $\bar{p} p$ and $p p$ Scattering at TeV Energies and Beyond, Nucl. Phys. B 299 (1988) 640 [INSPIRE].

[79] G. Audi, O. Bersillon, J. Blachot and A. Wapstra, The Nubase evaluation of nuclear and decay properties, Nucl. Phys. A 729 (2003) 3 [InSPIRE].

[80] C.J. Morningstar and M.J. Peardon, The Glueball spectrum from an anisotropic lattice study, Phys. Rev. D 60 (1999) 034509 [hep-lat/9901004] [INSPIRE].

[81] Y. Chen, A. Alexandru, S. Dong, T. Draper, I. Horváth et al., Glueball spectrum and matrix elements on anisotropic lattices, Phys. Rev. D 73 (2006) 014516 [hep-lat/0510074] [INSPIRE].

[82] H.B. Meyer, Glueball Regge trajectories, hep-lat/0508002 [INSPIRE].

[83] E. Gregory, A. Irving, B. Lucini, C. McNeile, A. Rago et al., Towards the glueball spectrum from unquenched lattice QCD, JHEP 10 (2012) 170 [arXiv:1208.1858] [INSPIRE].

[84] E. Meggiolaro, M. Giordano and N. Moretti, Asymptotic high-energy behavior of hadronic total cross sections from lattice QCD, arXiv:1304.3297 [INSPIRE].

[85] C. Ewerz, M. Maniatis and O. Nachtmann, A Model for Soft High-Energy Scattering: Tensor Pomeron and Vector Odderon, Annals Phys. 342 (2014) 31 [arXiv:1309.3478] [INSPIRE]. 